\title{
Pacific
}

Journal of

Mathematics

\section{KNOT COLOURING POLYNOMIALS}

\author{
Michael EISERMANN
}




\title{
KNOT COLOURING POLYNOMIALS
}

\author{
MiCHAEL EISERMANN
}

We introduce a natural extension of the colouring numbers of knots, called colouring polynomials, and study their relationship to Yang-Baxter invariants and quandle 2-cocycle invariants.

For a knot $K$ in the 3-sphere, let $\pi_{K}$ be the fundamental group of the knot complement $\mathbb{S}^{3} \backslash K$, and let $m_{K}, l_{K} \in \pi_{K}$ be a meridian-longitude pair. Given a finite group $G$ and an element $x \in G$ we consider the set of representations $\rho: \pi_{K} \rightarrow G$ with $\rho\left(m_{K}\right)=x$ and define the colouring polynomial as $\sum_{\rho} \rho\left(l_{K}\right)$. The resulting invariant maps knots to the group ring $\mathbb{Z} G$. It is multiplicative with respect to connected sum and equivariant with respect to symmetry operations of knots. Examples are given to show that colouring polynomials distinguish knots for which other invariants fail, in particular they can distinguish knots from their mutants, obverses, inverses, or reverses.

We prove that every quandle 2-cocycle state-sum invariant of knots is a specialization of some knot colouring polynomial. This provides a complete topological interpretation of these invariants in terms of the knot group and its peripheral system. Furthermore, we show that the colouring polynomial can be presented as a Yang-Baxter invariant, i.e. as the trace of some linear braid group representation. This entails that Yang-Baxter invariants can detect noninversible and nonreversible knots.

\section{Introduction and statement of results}

To each knot $K$ in the 3 -sphere $\mathbb{S}^{3}$ we can associate its knot group, that is, the fundamental group of the knot complement, denoted by

$$
\pi_{K}:=\pi_{1}\left(\mathbb{S}^{3} \backslash K\right) .
$$

This group is already a very strong invariant: it classifies unoriented prime knots [Whitten 1987; Gordon and Luecke 1989]. In order to capture the complete information, we consider a meridian-longitude pair $m_{K}, l_{K} \in \pi_{K}$ : the group system

MSC2000: primary 57M25; secondary 57M27.

Keywords: fundamental group of a knot, peripheral system, knot group homomorphism, quandle

2-cocycle state-sum invariant, Yang-Baxter invariant. 
$\left(\pi_{K}, m_{K}, l_{K}\right)$ classifies oriented knots in the 3 -sphere [Waldhausen 1968]. In particular, the group system allows us to tackle the problem of detecting asymmetries of a given knot (see Section 2C). Using this ansatz, M. Dehn [1914] proved that the two trefoil knots are chiral, and, half a century later, H. F. Trotter [1963] proved that pretzel knots are nonreversible. We will recover these results using knot colouring polynomials (see Section 2D).

Given a knot $K$, represented say by a planar diagram, we can easily read off the Wirtinger presentation of $\pi_{K}$ in terms of generators and relations (Section 3A). In general, however, such presentations are very difficult to analyze. As R. H. Crowell and R. H. Fox [1963, §VI.5] put it:

"What is needed are some standard procedures for deriving from a group presentation some easily calculable algebraic quantities which are the same for isomorphic groups and hence are so-called group invariants."

The classical approach is, of course, to consider abelian invariants, most notably the Alexander polynomial. In order to effectively extract nonabelian information, we consider the set of knot group homomorphisms $\operatorname{Hom}\left(\pi_{K} ; G\right)$ to some finite group $G$. The aim of this article is to organize this information and to generalize colouring numbers to colouring polynomials. In doing so, we will highlight the close relationship to Yang-Baxter invariants and their deformations on the one hand, and to quandle cohomology and associated state-sum invariants on the other hand.

From colouring numbers to colouring polynomials. A first and rather crude invariant is given by the total number of $G$-representations, denoted by

$$
F_{G}(K):=\left|\operatorname{Hom}\left(\pi_{K} ; G\right)\right| .
$$

This defines a map $F_{G}: \mathscr{K} \rightarrow \mathbb{Z}$ on the set $\mathscr{K}$ of isotopy classes of knots in $\mathbb{S}^{3}$. This invariant can be refined by further specifying the image of the meridian $m_{K}$, that is, we choose an element $x \in G$ and consider only those homomorphisms $\rho: \pi_{K} \rightarrow G$ satisfying $\rho\left(m_{K}\right)=x$. Their total number defines the knot invariant

$$
F_{G}^{x}(K):=\left|\operatorname{Hom}\left(\pi_{K}, m_{K} ; G, x\right)\right| .
$$

Example 1.1. Let $G$ be the dihedral group of order $2 p$, where $p \geq 3$ is odd, and let $x \in G$ be a reflection. Then $F_{G}^{x}$ is the number of $p$-colourings as introduced by Fox $[1962 ; 1970]$, here divided by $p$ for normalization such that $F_{G}^{x}(\bigcirc)=1$.

We will call $F_{G}^{x}$ the colouring number associated with $(G, x)$, in the dihedral case just as well as in the general case of an arbitrary group. Obviously $F_{G}$ can be recovered from $F_{G}^{x}$ by summation over all $x \in G$. In order to exploit the information of meridian and longitude, we introduce knot colouring polynomials as follows: 
Definition 1.2. Suppose that $G$ is a finite group and $x$ is one of its elements. The colouring polynomial $P_{G}^{x}: \mathscr{K} \rightarrow \mathbb{Z} G$ is defined as

$$
P_{G}^{x}(K):=\sum_{\rho} \rho\left(l_{K}\right),
$$

where the sum is taken over all homomorphisms $\rho: \pi_{K} \rightarrow G$ with $\rho\left(m_{K}\right)=x$.

By definition $P_{G}^{x}$ takes its values in the semiring $\mathbb{N} G$, but we prefer the more familiar group ring $\mathbb{Z} G \supset \mathbb{N} G$. We recover the colouring number $F_{G}^{x}=\varepsilon P_{G}^{x}$ by composing with the augmentation map $\varepsilon: \mathbb{Z} G \rightarrow \mathbb{Z}$. As it turns out, colouring polynomials allow us in a simple and direct manner to distinguish knots from their mirror images, as well as from their reverse or inverse knots. We will highlight some examples below.

Elementary properties. The invariant $P_{G}^{x}$ behaves very much like classical knot polynomials. Most notably, it nicely reflects the natural operations on knots: $P_{G}^{x}$ is multiplicative under connected sum and equivariant under symmetry operations (Section 2C).

Strictly speaking, $P_{G}^{x}(K)$ is, of course, not a polynomial but an element in the group ring $\mathbb{Z} G$. Since $l_{K}$ lies in the commutator subgroup $\pi_{K}^{\prime}$ and commutes with $m_{K}$, possible longitude images lie in the subgroup $\Lambda=C(x) \cap G^{\prime}$. Very often this subgroup will be cyclic, $\Lambda=\left\langle t \mid t^{n}=1\right\rangle$ say, in which case $P_{G}^{x}$ takes values in the truncated polynomial ring $\mathbb{Z} \Lambda=\mathbb{Z}[t] /\left(t^{n}\right)$. Here is a first and very simple example:

Example 1.3. We choose the alternating group $G=\mathrm{A}_{5}$ with basepoint $x=(12345)$. Here the longitude subgroup $\Lambda=\langle x\rangle$ is cyclic of order 5 . The colouring polynomials of the left- and right-handed trefoil knots are $1+5 x$ and $1+5 x^{-1}$ respectively, hence the trefoil knots are chiral. (There are five nontrivial colourings, one of which is shown in Section 3, Figure 5, and the other four are obtained by conjugating with $x$. This list is easily seen to be complete.)

Starting from scratch, i.e. from knot diagrams and Reidemeister moves, one usually appreciates Fox' notion of 3-colourability [1970] as the simplest proof of knottedness. In this vein, the preceding example is arguably one of the most elementary proofs of chirality, only rivalled by the Kauffman bracket leading to the Jones polynomial [Kauffman 1987].

Section 2D displays some further examples to show that colouring polynomials distinguish knots for which other invariants fail:

- They distinguish the Kinoshita-Terasaka knot from the Conway knot and show that none of them is inversible nor reversible nor obversible.

- They detect asymmetries of pretzel knots; thus, for example, they distinguish $B(3,5,7)$ from its inverse, reverse and obverse knot. 
- They distinguish the (inversible) knot $8_{17}$ from its reverse.

We also mention two natural questions that will not be pursued here:

Question 1.4. Can knot colouring polynomials detect other geometric properties of knots? Applications to periodic knots and ribbon knots would be most interesting.

Question 1.5. Do colouring polynomials distinguish all knots? Since the knot group system $\left(\pi_{K}, m_{K}, l_{K}\right)$ characterizes the knot $K$ [Waldhausen 1968, Cor. 6.5], and knot groups are residually finite [Thurston 1982, Thm.3.3], this question is not completely hopeless.

Colouring polynomials are Yang-Baxter invariants. Moving from empirical evidence to a more theoretical level, this article compares knot colouring polynomials with two other classes of knot invariants: Yang-Baxter invariants, derived from traces of Yang-Baxter representations of the braid group (Section 4), and quandle colouring state-sum invariants derived from quandle cohomology (Section 3). The result can be summarized as follows:

$\left\{\begin{array}{c}\text { Yang-Baxter } \\ \text { invariants }\end{array}\right\} \supset\left\{\begin{array}{c}\text { colouring } \\ \text { polynomials }\end{array}\right\} \supset\left\{\begin{array}{c}\text { quandle 2-cocycle } \\ \text { state-sum invariants }\end{array}\right\} \supset\left\{\begin{array}{c}\text { col. polynomials } \\ \text { with } \Lambda \text { abelian }\end{array}\right\}$

P. J. Freyd and D. N. Yetter [1989, Prop. 4.2.5] have shown that every colouring number $F_{G}^{x}: \mathscr{K} \rightarrow \mathbb{Z}$ can be obtained from a certain Yang-Baxter operator $c$ over $\mathbb{Z}$. We generalize this result to colouring polynomials:

Theorem 1.6 (Section 4C). Suppose that $G$ is a group with basepoint $x$ such that the subgroup $\Lambda=C(x) \cap G^{\prime}$ is abelian. Then the colouring polynomial $P_{G}^{x}$ : $\mathscr{K} \rightarrow \mathbb{Z} \Lambda$ is a Yang-Baxter invariant of closed knots: there exists a Yang-Baxter operator $\tilde{c}$ over the ring $\mathbb{Z} \Lambda$, such that the associated knot invariant coincides with (a constant multiple) of $P_{G}^{x}$.

In the general case, where $\Lambda$ is not necessarily abelian, Section 4B gives an analogous presentation of $P_{G}^{x}$ as a Yang-Baxter invariant of long knots (also called 1-tangles).

Corollary 1.7. Since $\Lambda$ is abelian in all our examples of Section $2 D$, it follows in particular that Yang-Baxter invariants can detect noninversible and nonreversible knots.

Remark 1.8. It follows from our construction that $\tilde{c}$ is a deformation of $c$ over the ring $\mathbb{Z} \Lambda$. Conversely, the deformation ansatz leads to quandle cohomology (see Section 4D). Elaborating this approach, M. Graña [2002] showed that quandle 2-cocycle state-sum invariants are Yang-Baxter invariants. The general theory of Yang-Baxter deformations of $c_{Q}$ over the power series ring $\mathbb{Q} \llbracket h \rrbracket$ has been developed in [Eisermann 2005]. 
Remark 1.9. The celebrated Jones polynomial and, more generally, all quantum invariants of knots, can be obtained from Yang-Baxter operators that are formal power series deformations of the trivial operator. This implies that the coefficients in this expansion are of finite type [Bar-Natan 1995, §2.1]. Part of their success lies in the fact that these invariants distinguish many knots, and in particular they easily distinguish mirror images. It is still unknown, however, whether finite type invariants can detect noninversible or nonreversible knots.

For colouring polynomials the construction is similar in that $P_{G}^{x}$ arises from a deformation of a certain operator $c$. There are, however, two crucial differences:

- The initial operator $c$ models conjugation (and is not the trivial operator),

- Its deformation $\tilde{c}$ is defined over $\mathbb{Z} \Lambda$ (and not over a power series ring).

As a consequence, the colouring polynomial $P_{G}^{x}$ is not of finite type, nor are its coefficients, nor any other real-valued invariant computed from it [Eisermann 2000b].

Quandle invariants are specialized colouring polynomials. A quandle, as introduced by D. Joyce [1982], is a set $Q$ with a binary operation whose axioms model conjugation in a group, or equivalently, the Reidemeister moves of knot diagrams. Quandles have been intensively studied by different authors and under various names; we review the relevant definitions in Section 3. The Lifting Lemma proved in Section 3B tells us how to pass from quandle to group colourings and back without any loss of information. On the level of knot invariants this implies the following result:

Theorem 1.10 (Section 3B). Every quandle colouring number $F_{Q}^{q}$ is the specialization of some knot colouring polynomial $P_{G}^{x}$.

Quandle cohomology was initially studied in order to construct invariants in low-dimensional topology: in [Carter et al. 1999; 2003b] it was shown how a 2cocycle $\lambda \in Z^{2}(Q, \Lambda)$ gives rise to a state-sum invariant of knots, $S_{Q}^{\lambda}: \mathscr{K} \rightarrow \mathbb{Z} \Lambda$, which refines the quandle colouring number $F_{Q}$. We prove the following result:

Theorem 1.11 (Section 3E). Every quandle 2-cocycle state-sum invariant of knots is the specialization of some knot colouring polynomial. More precisely, suppose that $Q$ is a connected quandle, $\Lambda$ is an abelian group, and $\lambda \in Z^{2}(Q, \Lambda)$ is a 2cocycle with associated invariant $S_{Q}^{\lambda}: \mathscr{K} \rightarrow \mathbb{Z} \Lambda$. Then there exists a group $G$ with basepoint $x$ and $a \mathbb{Z}$-linear map $\varphi: \mathbb{Z} G \rightarrow \mathbb{Z} \Lambda$ such that $S_{Q}^{\lambda}=\varphi P_{G}^{x} \cdot|Q|$.

This result provides a complete topological interpretation of quandle 2-cocycle state-sum invariants in terms of the knot group and its peripheral system. Conversely, we prove that state-sum invariants contain those colouring polynomials $P_{G}^{x}$ for which the longitude group $\Lambda=C(x) \cap G^{\prime}$ is abelian: 
Theorem 1.12 (Section 3D). Suppose that $G$ is a colouring group with basepoint $x$ such that the subgroup $\Lambda=C(x) \cap G^{\prime}$ is abelian. Then the colouring polynomial $P_{G}^{x}$ can be presented as a quandle 2-cocycle state-sum invariant. More precisely, the quandle $Q=x^{G}$ admits a 2-cocycle $\lambda \in Z^{2}(Q, \Lambda)$ such that $S_{Q}^{\lambda}=P_{G}^{x} \cdot|Q|$.

How this article is organized. Section 2 recalls the necessary facts about the knot group and its peripheral system. It then discusses connected sum and symmetry operations with respect to knot colouring polynomials and displays some applications. The main purpose is to give some evidence as to the scope and the usefulness of these invariants.

In Section 3 we examine quandle colourings and explain how to replace quandle colourings by group colourings without any loss of information. The correspondence between quandle extensions and quandle cohomology is then used to show how quandle 2-cocycle state-sum invariants can be seen as specializations of colouring polynomials.

Section 4 relates colouring polynomials with Yang-Baxter invariants. After recalling the framework of linear braid group representations, we show how colouring polynomials can be seen as Yang-Baxter deformations of colouring numbers.

\section{Knot groups and colouring polynomials}

This section collects basic facts about the knot group and its peripheral system (Section 2A) and their homomorphic images (Section 2B). We explain how the connected sum and symmetry operations affect the knot group system and how this translates to colouring polynomials (Section 2C). We then display some examples showing that colouring polynomials are a useful tool in distinguishing knots where other invariants fail (Section 2D).

2A. Peripheral system. We use fairly standard notation, which we recall from [Eisermann 2003] for convenience. A knot is a smooth embedding $k: \mathbb{S}^{1} \hookrightarrow \mathbb{S}^{3}$, considered up to isotopy. This is equivalent to considering the oriented image $K=$ $k\left(\mathbb{S}^{1}\right)$ in $\mathbb{S}^{3}$, again up to isotopy. A framing of $k$ is an embedding $f: \mathbb{S}^{1} \times \mathbb{D}^{2} \hookrightarrow \mathbb{S}^{3}$ such that $\left.f\right|_{\mathbb{S}^{1} \times 0}=k$. As basepoint of the space $\mathbb{S}^{3} \backslash K$ we choose $p=f(1,1)$. In the fundamental group $\pi_{K}:=\pi_{1}\left(\mathbb{S}^{3} \backslash K, p\right)$ we define the meridian $m_{K}=\left[\left.f\right|_{1 \times \mathbb{S}^{1}}\right]$ and the longitude $l_{K}=\left[\left.f\right|_{\mathbb{S}^{1} \times 1}\right]$. Up to isotopy the framing is characterized by the linking numbers $\operatorname{lk}\left(K, m_{K}\right) \in\{ \pm 1\}$ and $\operatorname{lk}\left(K, l_{K}\right) \in \mathbb{Z}$, and all combinations are realized. We will exclusively work with the standard framing, characterized by the linking numbers $\mathrm{lk}\left(K, m_{K}\right)=+1$ and $\mathrm{lk}\left(K, l_{K}\right)=0$.

Up to isomorphism, the triple $\left(\pi_{K}, m_{K}, l_{K}\right)$ is a knot invariant, and even a complete invariant: two knots $K$ and $K^{\prime}$ are isotopic if and only if there is a group isomorphism $\phi: \pi_{K} \rightarrow \pi_{K^{\prime}}$ with $\phi\left(m_{K}\right)=m_{K^{\prime}}$ and $\phi\left(l_{K}\right)=l_{K^{\prime}}$. This is a special 
case of Waldhausen's theorem on sufficiently large 3-manifolds; see [Waldhausen 1968, Cor. 6.5] and [Burde and Zieschang 1985, §3C].

Besides closed knots $k: \mathbb{S}^{1} \hookrightarrow \mathbb{S}^{3}$ it will be useful to consider long knots (also called 1-tangles), i.e. smooth embeddings $\ell: \mathbb{R} \hookrightarrow \mathbb{R}^{3}$ such that $\ell(t)=(t, 0,0)$ for all parameters $t$ outside of some compact interval. See [Eisermann 2003] for a detailed discussion with respect to knot groups and quandles.

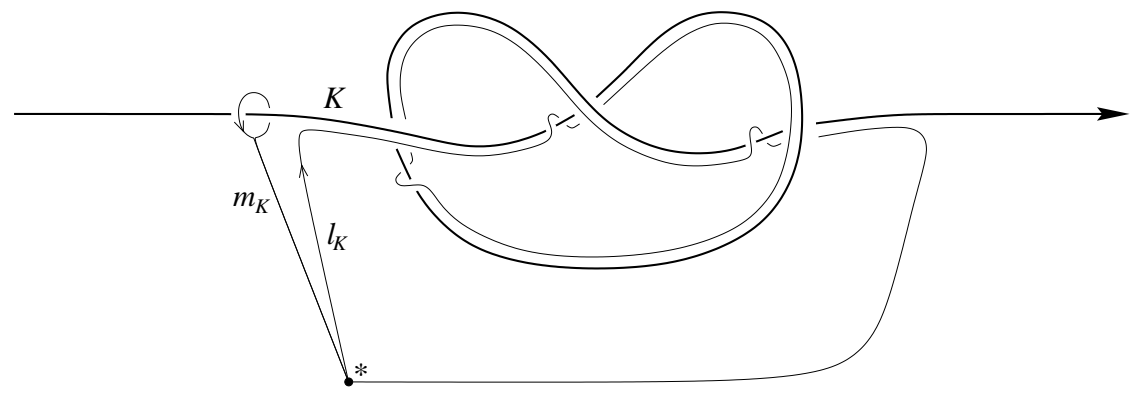

Figure 1. Meridian and longitude of a long knot.

2B. Colouring groups. Since knot groups are residually finite [Thurston 1982, Thm. 3.3], there are plenty of finite knot group representations. But which groups do actually occur as homomorphic images of knot groups? This question was raised by L. P. Neuwirth [1965], and first solved by F. González-Acuña:

Theorem 2.1 [González-Acuña 1975; Johnson 1980]. A pointed group $(G, x)$ is the homomorphic image of some knot group $\left(\pi_{K}, m_{K}\right)$ if and only if $G$ is finitely generated and $G=\left\langle x^{G}\right\rangle$.

The condition is necessary, because every knot group $\pi_{K}$ is finitely generated by conjugates of the meridian $m_{K}$. (See the Wirtinger presentation, recalled in Section 3A.) For a proof of sufficiency we refer to the article of D. Johnson [1980], who has found an elegant and ingeniously simple way to construct a knot $K$ together with an epimorphism $\left(\pi_{K}, m_{K}\right) \rightarrow(G, x)$. Here we restrict attention to finite groups:

Definition 2.2. Let $G$ be a finite group and $x \in G$. The pair $(G, x)$ is called a colouring group if the conjugacy class $x^{G}$ generates the whole group $G$. For example, every finite simple group $G$ is a colouring group with respect to any of its nontrivial elements $x \neq 1$.

Remark 2.3. Given a finite group $G_{0}$ and $x \in G_{0}$, every homomorphism

$$
\left(\pi_{K}, m_{K}\right) \rightarrow\left(G_{0}, x\right)
$$

maps to the subgroup $G_{1}:=\left\langle x^{G_{0}}\right\rangle$. If $G_{1}$ is strictly smaller than $G_{0}$, then we can replace $G_{0}$ by $G_{1}$. Continuing like this, we obtain a descending chain $G_{0} \supset$ 
$G_{1} \supset G_{2} \supset \cdots$, recursively defined by $G_{i+1}=\left\langle x^{G_{i}}\right\rangle$. Since $G_{0}$ is finite, this chain must stabilize, and we end up with a colouring group $G_{n}=\left\langle x^{G_{n}}\right\rangle$. Hence, we can assume without loss of generality that $(G, x)$ is a colouring group.

Given $(G, x)$ let $\Lambda^{*}$ be the set of longitude images $\rho\left(l_{K}\right)$, where $\rho$ ranges over all knot group homomorphisms $\rho:\left(\pi_{K}, m_{K}\right) \rightarrow(G, x)$ and all knots $K$. Then $\Lambda^{*}$ is a subgroup of $G$ [Johnson and Livingston 1989]. Since meridian $m_{K} \in \pi_{K}$ and longitude $l_{K} \in \pi_{K}^{\prime}$ commute, $\Lambda^{*}$ is contained in the subgroup $\Lambda=C(x) \cap G^{\prime}$, which will play an important rôle in subsequent arguments.

D. Johnson and C. Livingston [1989] have worked out a complete characterization of the subgroup $\Lambda^{*}$ in terms of homological obstructions. As an application, consider a colouring group $(G, x)$ that is perfect, i.e. $G^{\prime}=G$, and has cyclic centralizer, say $C(x)=\langle x\rangle$. The main result of [Johnson and Livingston 1989] affirms that $\Lambda^{*}=\Lambda=C(x)$. All of our examples in Section 2D are of this type.

2C. Knot and group symmetries. The knot group $\pi_{K}$ is obviously independent of orientations. In order to define the longitude, however, we have to specify the orientation of $K$, and the definition of the meridian additionally depends on the orientation of $\mathbb{S}^{3}$. Changing these orientations defines the following symmetry operations:

Definition 2.4. Let $K \subset \mathbb{S}^{3}$ be an oriented knot. The same knot with the opposite orientation of $\mathbb{S}^{3}$ is the mirror image or the obverse of $K$, denoted $K^{\times}$. (We can represent this as $K^{\times}=\sigma K$, where $\sigma: \mathbb{S}^{3} \rightarrow \mathbb{S}^{3}$ is a reflection.) Reversing the orientation of the knot $K$ yields the reverse knot $K^{!}$. Inverting both orientations yields the inverse knot $K^{*}$.

Please note that different authors use different terminology, in particular reversion and inversion are occasionally interchanged. Here we adopt the notation of J. H. Conway [1970].

Proposition 2.5. Let $K$ be an oriented knot with group system

$$
\check{\pi}(K)=\left(\pi_{K}, m_{K}, l_{K}\right) .
$$

Obversion, reversion and inversion affect the group system as follows:

$$
\begin{aligned}
\check{\pi}\left(K^{\times}\right) & =\left(\pi_{K}, m_{K}^{-1}, l_{K}\right), \\
\check{\pi}\left(K^{!}\right) & =\left(\pi_{K}, m_{K}^{-1}, l_{K}^{-1}\right), \\
\check{\pi}\left(K^{*}\right) & =\left(\pi_{K}, m_{K}, l_{K}^{-1}\right) .
\end{aligned}
$$

The fundamental group of the connected sum $K \sharp L$ is the amalgamated product $\pi_{K} * \pi_{L}$ modulo $m_{K}=m_{L}$. Its meridian is $m_{K}$ and its longitude is $l_{K} l_{L}$. 
Corollary 2.6. Every colouring polynomial $P_{G}^{x}: \mathscr{K} \rightarrow \mathbb{Z} G$ is multiplicative, that is, we have $P_{G}^{x}(K \sharp L)=P_{G}^{x}(K) \cdot P_{G}^{x}(L)$ for any two knots $K$ and $L$.

In order to formulate the effect of inversion, let ${ }^{*}: \mathbb{Z} G \rightarrow \mathbb{Z} G$ be the linear extension of the inversion map $G \rightarrow G, g \mapsto g^{-1}$.

Corollary 2.7. Every colouring polynomial $P_{G}^{x}: \mathscr{K} \rightarrow \mathbb{Z} G$ is equivariant under inversion, i.e. $P_{G}^{x}\left(K^{*}\right)=P_{G}^{x}(K)^{*}$ for every knot $K$. In particular, the colouring number $F_{G}^{x}(K)$ is invariant under inversion of $K$.

Obversion and reversion of knots can similarly be translated into symmetries of colouring polynomials, but to do so we need a specific automorphism of $G$ :

Definition 2.8. An automorphism ${ }^{\times}: G \rightarrow G$ with $x^{\times}=x^{-1}$ is called an obversion of $(G, x)$. An antiautomorphism ${ }^{!}: G \rightarrow G$ with $x^{!}=x$ is called a reversion of $(G, x)$.

Obviously a group $(G, x)$ possesses a reversion if and only if it possesses an obversion. They are in general not unique, because they can be composed with any automorphism $\alpha \in \operatorname{Aut}(G, x)$, for example conjugation by an element in $C(x)$.

Remark 2.9. The braid group $B_{n}$, recalled in Section $4 \mathrm{~A}$ below, has a unique antiautomorphism $!: \mathrm{B}_{n} \rightarrow \mathrm{B}_{n}$ fixing the standard generators $\sigma_{1}, \ldots, \sigma_{n-1}$. Analogously there exists a unique automorphism ${ }^{\times}: \mathrm{B}_{n} \rightarrow \mathrm{B}_{n}$ mapping each standard generator $\sigma_{i}$ to its inverse $\sigma_{i}^{-1}$. The exponent sum $\mathrm{B}_{n} \rightarrow \mathbb{Z}$ shows that this cannot be an inner automorphism.

These symmetry operations on braids correspond to the above symmetry operations on knots: if a knot $K$ is represented as the closure of the braid $\beta$ (see Section $4 \mathrm{~A}$ ), then the inverse braid $\beta^{-1}$ represents the inverse knot $K^{*}$, the reverse braid $\beta^{!}$represents the reverse $\operatorname{knot} K^{!}$, and the obverse braid $\beta^{\times}$represents the obverse knot $K^{\times}$.

Given an obversion and a reversion of $(G, x)$, their linear extensions to the group ring $\mathbb{Z} G$ will also be denoted by ${ }^{\times}: \mathbb{Z} G \rightarrow \mathbb{Z} G$ and $!: \mathbb{Z} G \rightarrow \mathbb{Z} G$, respectively. We can now formulate the equivariance of the corresponding colouring polynomials:

Corollary 2.10. Suppose that $(G, x)$ possesses an obversion ${ }^{\times}$and a reversion! Then the colouring polynomial $P_{G}^{x}$ is equivariant with respect to obversion and reversion, that is, we have $P_{G}^{x}\left(K^{\times}\right)=P_{G}^{x}(K)^{\times}$and $P_{G}^{x}\left(K^{!}\right)=P_{G}^{x}(K)^{!}$for every $k n o t K$. In this case the colouring numbers of $K, K^{*}, K^{\times}, K^{!}$are the same.

Example 2.11. Every element $x$ in the symmetric group $S_{n}$ is conjugated to its inverse $x^{-1}$, because both have the same cycle structure. Any such conjugation defines an obversion $\left(\mathrm{S}_{n}, x\right) \rightarrow\left(\mathrm{S}_{n}, x^{-1}\right)$. This argument also applies to alternating groups: given $x \in \mathrm{A}_{n}$ we know that $x$ is conjugated to $x^{-1}$ in $\mathrm{S}_{n}$. Since $\mathrm{A}_{n}$ is normal in $\mathrm{S}_{n}$, this conjugation restricts to an obversion $\left(\mathrm{A}_{n}, x\right) \rightarrow\left(\mathrm{A}_{n}, x^{-1}\right)$. This need not be an inner automorphism. 
On the other hand, some groups do not permit any obversion at all:

Example 2.12. Let $\mathbb{F}$ be a finite field and let $G=\mathbb{F} \rtimes \mathbb{F}^{\times}$be its affine group. We have $\operatorname{Aut}(G)=\operatorname{Inn}(G) \rtimes \operatorname{Gal}(\mathbb{F})$, where $\operatorname{Gal}(\mathbb{F})$ is the Galois group of $\mathbb{F}$ over its prime field $\mathbb{F}_{p}$. If $\mathbb{F}=\mathbb{F}_{p}$, then every automorphism of $G$ is inner and thus induces the identity on the abelian quotient $\mathbb{F}^{\times}$. If $p \geq 5$, we can choose an element $x=(a, b) \in$ $G$ whose projection to $\mathbb{F}^{\times}$satisfies $b \neq b^{-1}$. Hence there is no automorphism of $G$ that maps $x$ to $x^{-1}$. Indeed, searching all groups of small order with GAP [2006], we find that the smallest group having this property is $\mathbb{F}_{5} \rtimes \mathbb{F}_{5}^{\times}$of order 20 .

For the sake of completeness we expound the following elementary result:

Proposition 2.13. The affine group $G=\mathbb{F} \rtimes \mathbb{F}^{\times}$satisfies $\operatorname{Aut}(G)=\operatorname{Inn}(G) \rtimes \operatorname{Gal}(\mathbb{F})$.

Proof. The product in $G$ is given by $(a, b)(c, d)=(a+b c, b d)$, and so Gal $(\mathbb{F})$ can be seen as a subgroup of $\operatorname{Aut}(G)$, where $\phi \in \operatorname{Gal}(\mathbb{F})$ acts as $(a, b) \mapsto(\phi(a), \phi(b))$. Since $\operatorname{Inn}(G)$ is a normal subgroup of $\operatorname{Aut}(G)$ with $\operatorname{Inn}(G) \cap \operatorname{Gal}(\mathbb{F})=\left\{\operatorname{id}_{G}\right\}$, we see that $\operatorname{Aut}(G)$ contains the semidirect product $\operatorname{Inn}(G) \rtimes \operatorname{Gal}(\mathbb{F})$.

It remains to show that every $\alpha \in \operatorname{Aut}(G)$ belongs to $\operatorname{Inn}(G) \rtimes \operatorname{Gal}(\mathbb{F})$. This is trivially true for $\mathbb{F}=\mathbb{F}_{2}$, so we will assume that $\mathbb{F}$ has more than two elements. It is then easily verified that $G^{\prime}=\mathbb{F} \times\{1\}$. Let $\zeta$ be a generator of the multiplicative group $\mathbb{F}^{\times}$. We have $\alpha(1,1)=(u, 1)$ with $u \in \mathbb{F}^{\times}$, and $\alpha(0, \zeta)=(v, \xi)$ with $v \in \mathbb{F}$, $\xi \in \mathbb{F}^{\times}, \xi \neq 1$. Conjugating by $w=\left(v(1-\xi)^{-1}, u\right)$, we obtain $(u, 1)^{w}=(1,1)$ and $(v, \xi)^{w}=(0, \xi)$. In the sequel we can thus assume $u=1$ and $v=0$. This implies $\alpha(0, b)=(0, \phi(b))$ with $\phi: \mathbb{F}^{\times} \rightarrow \mathbb{F}^{\times}, \zeta^{n} \mapsto \xi^{n}$ for all $n \in \mathbb{Z}$. Extending this by $\phi(0)=0$ we obtain a bijection $\phi: \mathbb{F} \rightarrow \mathbb{F}$ satisfying $\phi(a b)=\phi(a) \phi(b)$ for all $a, b \in \mathbb{F}$. Moreover, we find $\alpha(a, 1)=(\phi(a), 1)$ : this is clear for $a=0$, and for $a \neq 0$ we have $(a, 1)=(0, a)(1,1)$ and thus $\alpha(a, 1)=(0, \phi(a))(1,1)=(\phi(a), 1)$. This proves that $\phi(a+b)=\phi(a)+\phi(b)$ for all $a, b \in \mathbb{F}$, whence $\phi \in \operatorname{Gal}(\mathbb{F})$. We conclude that $\alpha(a, b)=(\phi(a), \phi(b))$, as claimed.

2D. Examples and applications. The preceding discussion indicates that symmetries of the group $(G, x)$ affect the colouring polynomial $P_{G}^{x}(K)$ just as well as symmetries of the knot $K$. We point out several examples:

Example 2.14. Let $p$ be a prime and let $G=\mathrm{PSL}_{2} \mathbb{F}_{p}$ be equipped with basepoint $z=\left[\begin{array}{ll}1 & 1 \\ 0 & 1\end{array}\right]$ of order $p$. Inversion, obversion, and reversion are realized by

$$
\left[\begin{array}{ll}
a & b \\
c & d
\end{array}\right]^{*}=\left[\begin{array}{rr}
d & -b \\
-c & a
\end{array}\right], \quad\left[\begin{array}{ll}
a & b \\
c & d
\end{array}\right]^{\times}=\left[\begin{array}{rr}
a & -b \\
-c & d
\end{array}\right], \quad\left[\begin{array}{ll}
a & b \\
c & d
\end{array}\right]^{!}=\left[\begin{array}{ll}
d & b \\
c & a
\end{array}\right] .
$$

We have $C(z)=\langle z\rangle$. For $p=2$ and $p=3$ one finds that the longitude group $\Lambda=C(z) \cap G^{\prime}$ is trivial. For $p \geq 5$ the group $G$ is perfect (even simple), hence $\Lambda=\langle z\rangle$. We conclude that the colouring polynomial $P_{G}^{z}$ is insensitive to reversion: we have $P_{G}^{z}(K) \in \mathbb{Z}\langle z\rangle$ and reversion fixes $z$ and therefore all elements in $\mathbb{Z}\langle z\rangle$. 
Example 2.15. Consider an alternating group $G=\mathrm{A}_{n}$ with $n \geq 3$, and a cycle $x=(123 \ldots l)$ of maximal length, that is, $l=n$ for $n$ odd and $l=n-1$ for $n$ even. As we have pointed out above, a suitable conjugation in $\mathrm{S}_{n}$ produces an obversion $(G, x) \rightarrow\left(G, x^{-1}\right)$. We have $C(x)=\langle x\rangle$. For $n=3$ and $n=4$ one finds that the longitude group $\Lambda=C(x) \cap G^{\prime}$ is trivial. For $n \geq 5$ the group $G$ is perfect (even simple), hence the longitude group is $\Lambda=\langle x\rangle$. Again we conclude that the colouring polynomial $P_{G}^{x}$ is insensitive to reversion.

We observe that for $l=3,7,11, \ldots$ an obversion of $(G, x)$ cannot be realized by an inner automorphism: consider for example $G=\mathrm{A}_{11}$ and $x=$ (abcdefghijk): in $\mathrm{S}_{11}$ the centralizer is $C(x)=\langle x\rangle$ and consequently every permutation $\sigma \in \mathrm{S}_{11}$ with $x^{\sigma}=x^{-1}$ is of the form $\sigma=x^{k}(\mathrm{ak})(\mathrm{bj})(\mathrm{ci})(\mathrm{dh})(\mathrm{eg})$ and thus odd. The same argument shows that for $n=5,9,13, \ldots$ an obversion of $(G, x)$ can be realized by an inner automorphism.

Example 2.16. As a more exotic example, let us finally consider the Mathieu group $M_{11}$, i.e. the unique simple group of order $7920=2^{4} \cdot 3^{2} \cdot 5 \cdot 11$, and the smallest of the sporadic simple groups [Conway et al. 1985]. It can be presented as a subgroup of $\mathrm{A}_{11}$, for example as

$$
G=\langle x, y\rangle \text { with } \quad x=(\text { abcdefghijk }), y=(\text { abcejikdghf }) .
$$

This presentation has been obtained from GAP and can easily be verified with any group-theory software by checking that $G$ is simple of order 7920. The Mathieu group $M_{11}$ is particularly interesting for us, because it does not allow an obversion. To see this it suffices to know that its group of outer automorphisms is trivial, in other words, every automorphism of $M_{11}$ is realized by conjugation. In $M_{11}$ the element $x$ is not conjugated to its inverse - this is not even possible in $A_{11}$ according to the preceding example. Hence there is no automorphism of $M_{11}$ that maps $x$ to $x^{-1}$.

Applied to colouring polynomials, this means that there is a priori no restriction on the invariants of a knot and its mirror image. As a concrete example we consider the Kinoshita-Terasaka knot $K$ and the Conway knot $C$ displayed in Figure 2.
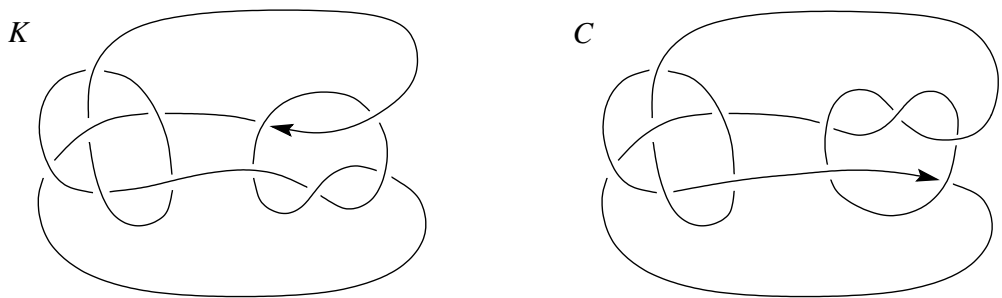

Figure 2. The Kinoshita-Terasaka knot and the Conway knot. 
Both knots have trivial Alexander polynomial. They differ only by rotation of a 2-tangle, in other words they are mutants in the sense of Conway [1970]. Therefore neither the Jones, HOMFLYPT nor Kauffman polynomial can distinguish between $K$ and $C$, see [Lickorish 1997]. With the help of a suitable colouring polynomial the distinction is straightforward:

Example 2.17. R. Riley [1971] has studied knot group homomorphisms to the simple group $G=\mathrm{PSL}_{2} \mathbb{F}_{7}$ of order 168 . Let $z$ be an element of order 7 , say $z=\left[\begin{array}{ll}1 & 1 \\ 0 & 1\end{array}\right]$. Then the associated colouring polynomials are

$$
\begin{aligned}
& P_{G}^{z}(K)=P_{G}^{z}(C)=1+7 z^{5}+7 z^{6}, \\
& P_{G}^{z}\left(K^{*}\right)=P_{G}^{z}\left(C^{*}\right)=1+7 z+7 z^{2} .
\end{aligned}
$$

This shows that both knots are chiral. By a more detailed analysis of their coverings, Riley could even show that $K$ and $C$ are distinct.

Example 2.18. To distinguish $K$ and $C$ we give a simple and direct argument using colouring polynomials. For every element $x \in \mathrm{PSL}_{2} \mathbb{F}_{7}$ of order 3 , say $x=\left[\begin{array}{rr}0 & 1 \\ -1 & 1\end{array}\right]$, the associated colouring polynomial distinguishes $K$ and $C$ :

$$
\begin{array}{ll}
P_{G}^{x}(K)=1+6 x, & P_{G}^{x}(C)=1+12 x, \\
P_{G}^{x}\left(K^{*}\right)=1+6 x^{2}, & P_{G}^{x}\left(C^{*}\right)=1+12 x^{2} .
\end{array}
$$

Both invariants, $P_{G}^{z}$ and $P_{G}^{x}$, show chirality but are insensitive to reversion.

These and the following colouring polynomials were calculated with the help of an early prototype of the computer program KnotGRep, an ongoing programming project to efficiently construct the set of knot group homomorphisms to a finite group. Even though general-purpose software may be less comfortable, our results can also be obtained from the Wirtinger presentation (Section 3A) using GAP or similar group-theoretic software.

Example 2.19. The alternating group $G=\mathrm{A}_{7}$ with basepoint $x=(1234567)$ yields

$$
\begin{array}{ll}
P_{G}^{x}(K)=1+7 x^{2}+28 x^{5}+28 x^{6}, & P_{G}^{x}(C)=1+7 x^{2}+7 x^{3}+21 x^{5}+14 x^{6} \\
P_{G}^{x}\left(K^{*}\right)=1+28 x+28 x^{2}+7 x^{5}, & P_{G}^{x}\left(C^{*}\right)=1+14 x+21 x^{2}+7 x^{4}+7 x^{5}
\end{array}
$$

Again this invariant distinguishes $K$ et $C$ and shows their chirality, but is insensitive to reversion, as explained in Example 2.15 above.

Example 2.20. More precise information can be obtained using the Mathieu group $M_{11}$, presented as the permutation group $(G, x)$ in Example 2.16 above. For the 
Kinoshita-Terasaka knot $K$ and the Conway knot $C$ one finds

$$
\begin{array}{ll}
P_{G}^{x}(K)=1+11 x^{3}+11 x^{7}, & P_{G}^{x}(C)=1+11 x^{3}+11 x^{7}, \\
P_{G}^{x}\left(K^{*}\right)=1+11 x^{4}+11 x^{8}, & P_{G}^{x}\left(C^{*}\right)=1+11 x^{4}+11 x^{8}, \\
P_{G}^{x}\left(K^{\times}\right)=1+11 x^{4}+22 x^{8}, & P_{G}^{x}\left(C^{\times}\right)=1+11 x^{4}+11 x^{6}+11 x^{8}, \\
P_{G}^{x}\left(K^{!}\right)=1+22 x^{3}+11 x^{7}, & P_{G}^{x}\left(C^{!}\right)=1+11 x^{3}+11 x^{5}+11 x^{7} .
\end{array}
$$

Consequently all eight knots are distinct; $K$ and $C$ are neither inversible nor obversible nor reversible. (This example was inspired by G. Kuperberg [1996], who used the colouring number $F_{G}^{x}$ to distinguish the knot $C$ from its reverse $C^{!}$.)

Usually it is very difficult to detect nonreversibility of knots. Most invariants fail to do so, including the usual knot polynomials. In view of the simplicity of our approach, the success of knot colouring polynomials is remarkable. We give two further examples:

Example 2.21. The family of pretzel knots $B\left(p_{1}, p_{2}, p_{3}\right)$, parametrized by odd integers $p_{1}, p_{2}, p_{3}$, is depicted in Figure 3, left. According to the classification of pretzel knots (see [Burde and Zieschang 1985], §12), the pretzel knot $B=$ $B(3,5,7)$ is neither reversible nor obversible nor inversible. For the Mathieu group $G=M_{11}$ with basepoint $x$ as in Example 2.20 we obtain

$$
\begin{array}{ll}
P_{G}^{x}(B)=1+11 x, & P_{G}^{x}\left(B^{\times}\right)=1+11 x^{7}, \\
P_{G}^{x}\left(B^{*}\right)=1+11 x^{10}, & P_{G}^{x}\left(B^{!}\right)=1+11 x^{4} .
\end{array}
$$

Again the colouring polynomial shows that the knot $B$ possesses none of the three symmetries. Historically, pretzel knots were the first examples of nonreversible knots. Their nonreversibility was first proven by H. F. Trotter [1963] by representing the knot group system on a suitable triangle group acting on the hyperbolic plane.
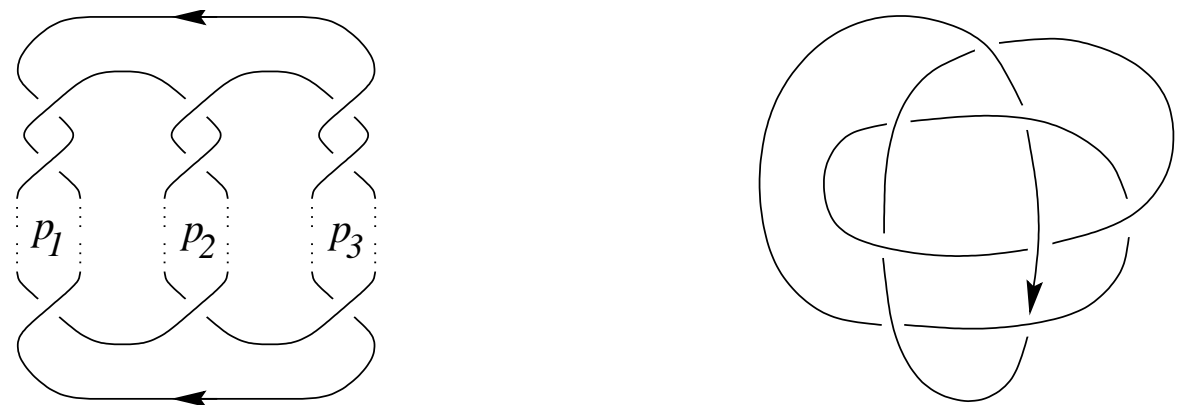

Figure 3. Left: the pretzel knot $B\left(p_{1}, p_{2}, p_{3}\right)$. Right: the knot $8_{17}$. 
Example 2.22. Figure 3, right, shows the knot 817 , which is the smallest nonreversible knot. It is a 3-bridge knot but not a pretzel knot, and there is no general classification theorem available. To analyze this example we choose once more the Mathieu group $M_{11}$ with basepoint $x$ as above. The knot $8_{17}$ then has colouring polynomial $1+11 x^{5}+11 x^{6}$ whereas the reverse knot has trivial colouring polynomial 1. (Here even the colouring number $F_{G}^{x}$ suffices to prove that this knot is nonreversible.) We remark that $8_{17}$ is inversible and that this symmetry is reflected in the symmetry of its colouring polynomials.

The colouring polynomial $P_{G}^{x}(K)$ is, by definition, an element in the group ring $\mathbb{Z} G$, and it actually lies in the much smaller ring $\mathbb{Z} \Lambda$. The following symmetry consideration further narrows down the possible values. It is included here to explain one of the observations that come to light in the previous examples, but it will not be used in the sequel.

Proposition 2.23. Let $(G, x)$ be a colouring group. If conjugation by $x$ has order $p^{k}$ for some prime $p$, then the colouring polynomial satisfies $P_{G}^{x}(K) \equiv 1(\bmod p)$.

Proof. The cyclic subgroup $\langle x\rangle$ acts on the set $\operatorname{Hom}\left(\pi_{K}, m_{K} ; G, x\right)$ by conjugation. The only fixed point is the trivial representation $\left(\pi(K), m_{K}\right) \rightarrow(\mathbb{Z}, 1) \rightarrow$ $(G, x)$. This can be most easily seen by interpreting group homomorphisms $\rho$ : $\left(\pi_{K}, m_{K}\right) \rightarrow(G, x)$ as colourings $f:(D, 0) \rightarrow(G, x)$ of a knot diagram $D$, see Section 3A below. If $f^{x}=f$ then all colours of $f$ commute with $x$ : following the diagram from the first to the last arc we see by induction that all colours are in fact equal to $x$. Since there is only one component, we conclude that $f$ is the trivial colouring, corresponding to the trivial representation.

Every nontrivial representation $\rho$ appears in an orbit of length $p^{\ell}$, for some integer $\ell \geq 1$. Since $\rho\left(l_{K}\right)$ commutes with $x$, all representations in such an orbit have the same longitude image in $G$. The sum $P_{G}^{x}(K)$ thus begins with 1 for the trivial representation, and all other summands can be grouped to multiples of $p$.

\section{Quandle invariants are specialized colouring polynomials}

The Wirtinger presentation allows us to interpret knot group homomorphisms as colourings of knot diagrams. Since such colourings involve only conjugation, they are most naturally treated in the category of quandles, as introduced by Joyce [1982]. We recall the basic definitions concerning quandles and quandle colourings in Section 3A, and explain in Section 3B how to pass from quandles to groups and back without any loss of information.

Quandle cohomology was studied in [Carter et al. 1999; 2003b], where it was shown how a 2-cocycle gives rise to a state-sum invariant of knots in $\mathbb{S}^{3}$. We recall this construction in Section 3D and show that every colouring polynomial $P_{G}^{x}$ can 
be presented as a quandle 2-cocycle state-sum invariant, provided that the subgroup $\Lambda=C(x) \cap G^{\prime}$ is abelian (Theorem 3.24).

In order to prove the converse, we employ the cohomological classification of central quandle extensions established in [Eisermann 2003; Carter et al. 2003a], recalled in Section $3 \mathrm{C}$ below. This allows us to prove in Section $3 \mathrm{E}$ that every quandle 2-cocycle state-sum invariant is the specialization of a suitable knot colouring polynomial (Theorem 3.25).

3A. Wirtinger presentation, quandles, and colourings. Our exposition follows [Eisermann 2003], to which we refer for further details. We consider a long knot diagram as in Figure 1 and number the arcs consecutively from 0 to $n$. At the end of arc number $i-1$, we undercross arc number $\kappa i=\kappa(i)$ and continue on arc number $i$. We denote by $\varepsilon i=\varepsilon(i)$ the sign of this crossing, as depicted in Figure 4 . The maps $\kappa:\{1, \ldots, n\} \rightarrow\{0, \ldots, n\}$ and $\varepsilon:\{1, \ldots, n\} \rightarrow\{ \pm 1\}$ are the Wirtinger code of the diagram.

Theorem 3.1. Suppose that a knot $L$ is represented by a long knot diagram with Wirtinger code $(\kappa, \varepsilon)$ as above. Then the knot group allows the presentation

$$
\pi_{L}=\left\langle x_{0}, x_{1}, \ldots, x_{n} \mid r_{1}, \ldots, r_{n}\right\rangle \text { with relation } r_{i} \text { being } x_{i}=x_{\kappa i}^{-\varepsilon i} x_{i-1} x_{\kappa i}^{\varepsilon i} \text {. }
$$

As peripheral system we can choose $m_{L}=x_{0}$ and $l_{L}=\prod_{i=1}^{i=n} x_{i-1}^{-\varepsilon i} x_{\kappa i}^{\varepsilon i}$.

For a proof see [Crowell and Fox 1963, §VI.3] or [Burde and Zieschang 1985, $\S 3 \mathrm{~B}]$. The Wirtinger presentation works just as well for a closed knot diagram. Since $\operatorname{arcs} 0$ and $n$ are then identified, this amounts to adding the (redundant) relation $x_{0}=x_{n}$ to the above presentation. The group is, of course, the same.

The Wirtinger presentation allows us to interpret knot group homomorphisms $\pi_{L} \rightarrow G$ as colourings. More precisely, a $G$-colouring of the diagram $D$ is a map $f:\{0, \ldots, n\} \rightarrow G$ such that $f(i)=f(\kappa i)^{-\varepsilon i} f(i-1) f(\kappa i)^{\varepsilon i}$. In other words, at each coloured crossing as in Figure 4 the colours $a$ and $c$ are conjugated via $a^{b}=c$. Such a colouring is denoted by $f: D \rightarrow G$. We denote by $\operatorname{Col}(D ; G)$ the set of colourings of $D$ with colours in $G$. For a long knot diagram $D$, we denote by $\operatorname{Col}(D, 0 ; G, x)$ the subset of colourings that colour arc number 0 with colour $x$. The Wirtinger presentation establishes natural bijections $\operatorname{Hom}\left(\pi_{K} ; G\right) \cong$ $\operatorname{Col}(D ; G)$ and $\operatorname{Hom}\left(\pi_{K}, m_{K} ; G, x\right) \cong \operatorname{Col}(D, 0 ; G, x)$.

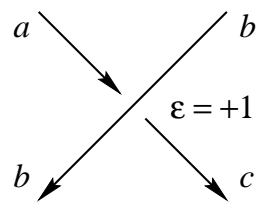

$$
\begin{aligned}
& a * b=c \\
& c * b=a
\end{aligned}
$$

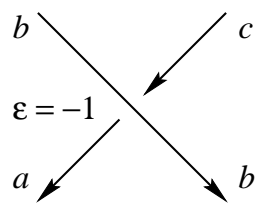

Figure 4. Wirtinger rules for colouring a knot diagram. 
Example 3.2. Figure 5 shows a colouring of the left-handed trefoil knot (represented as a long knot) with elements in the alternating group $\mathrm{A}_{5}$. Note that all definitions readily extend to closed knot diagrams.

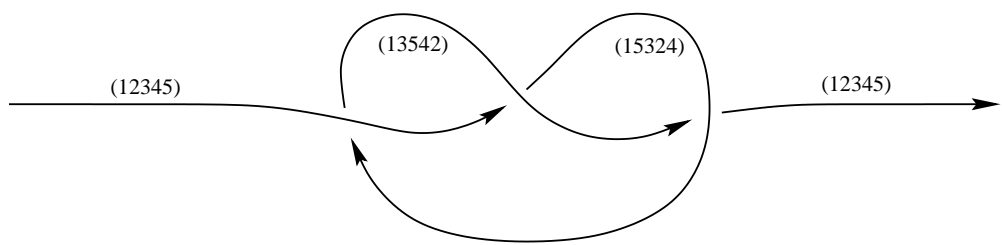

Figure 5. A5-colouring of the left-handed trefoil knot.

The Wirtinger presentation of $\pi_{K}$ involves only conjugation but not the group multiplication itself. The underlying algebraic structure can be described as follows:

Definition 3.3. A quandle is a set $Q$ with two binary operations $*, \bar{*}: Q \times Q \rightarrow Q$ satisfying the following axioms for all $a, b, c \in Q$ :

(Q1) $a * a=a$

(Q2) $(a * b) \mp b=(a \bar{*}) * b=a$

(Q3) $(a * b) * c=(a * c) *(b * c)$ (idempotency), (right invertibility), (self-distributivity).

As already mentioned, the notion (and name) was introduced in [Joyce 1982]. The same notion was studied by S. V. Matveev [1982] under the name "distributive groupoid", and by Kauffman [2001] who called it "crystal". Quandle axioms (Q2) and (Q3) are equivalent to saying that for every $b \in Q$ the right translation $\varrho_{b}: a \mapsto$ $a * b$ is an automorphism of $Q$. Such structures were called "automorphic sets" by E. Brieskorn [1988]. The somewhat shorter term rack was preferred by R. Fenn and C. P. Rourke [1992]. The notion has been generalized to "crossed $G$-sets" by Freyd and Yetter [1989].

Definition 3.4. As before, let $D$ be a long knot diagram, its arcs being numbered by $0, \ldots, n$. A $Q$-colouring, denoted $f: D \rightarrow Q$, is a map $f:\{0, \ldots, n\} \rightarrow Q$ such that at each crossing as in Figure 4 the three colours $a, b, c$ satisfy the relation $a * b=c$. We denote by $\operatorname{Col}(D ; Q)$ the set of $Q$-colourings, and by $\operatorname{Col}(D, 0 ; Q, q)$ the subset of colourings satisfying $f(0)=q$.

Proposition 3.5 [Joyce 1982]. The quandle axioms ensure that each Reidemeister move $D \leftrightharpoons D^{\prime}$ induces bijections $\operatorname{Col}(D ; Q) \leftrightharpoons \operatorname{Col}\left(D^{\prime} ; Q\right)$ and $\operatorname{Col}(D, 0 ; Q, q) \leftrightharpoons$ $\operatorname{Col}\left(D^{\prime}, 0 ; Q, q\right)$. In particular, if $Q$ is finite, then the colouring numbers $F_{Q}(D)=$ $|\operatorname{Col}(D ; Q)|$ and $F_{Q}^{q}(D)=|\operatorname{Col}(D, 0 ; Q, q)|$ are knot invariants. 
3B. From quandle colourings to group colourings and back. In many respects quandles are close to groups. For colourings we will now explain how to pass from quandles to groups and back without any loss of information.

Definition 3.6. A quandle homomorphism is a map $\phi: Q \rightarrow Q^{\prime}$ that satisfies $\phi(a * b)=\phi(a) * \phi(b)$, and hence $\phi(a \bar{*} b)=\phi(a) \bar{*} \phi(b)$, for all $a, b \in Q$.

Definition 3.7. The automorphism group $\operatorname{Aut}(Q)$ consists of all bijective homomorphisms $\phi: Q \rightarrow Q$. We adopt the convention that automorphisms of $Q$ act on the right, written $a^{\phi}$, which means that their composition $\phi \psi$ is defined by $a^{(\phi \psi)}=\left(a^{\phi}\right)^{\psi}$ for all $a \in Q$.

Definition 3.8. The group $\operatorname{Inn}(Q)=\left\langle\varrho_{b} \mid b \in Q\right\rangle$ of inner automorphisms is the subgroup of $\operatorname{Aut}(Q)$ generated by all right translations $\varrho_{b}: a \mapsto a * b$. The quandle $Q$ is called connected if the action of $\operatorname{Inn}(Q)$ on $Q$ is transitive.

In view of the map $\varrho: Q \rightarrow \operatorname{Inn}(Q), b \mapsto \varrho_{b}$, we also write $a^{b}=a * b$ for the operation in a quandle. Conversely, it will sometimes be convenient to write $a * b=b^{-1} a b$ for the conjugation in a group. In neither case will there be any danger of confusion.

Definition 3.9. A representation of a quandle $Q$ on a group $G$ is a map $\phi: Q \rightarrow G$ such that $\phi(a * b)=\phi(a) * \phi(b)$ for all $a, b \in Q$. In other words, the following diagram commutes:

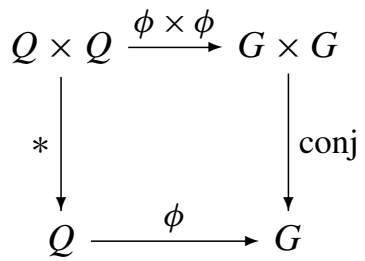

For example, the natural map $\varrho: Q \rightarrow \operatorname{Aut}(Q)$ satisfies $\varrho(a * b)=\varrho(a) * \varrho(b)$. We call $\varrho$ the inner representation of $Q$. Moreover it satisfies $\varrho\left(a^{g}\right)=\varrho(a)^{g}$ for all $a \in Q$ and $g \in \operatorname{Aut}(Q)$. This is the prototype of an augmentation:

Definition 3.10. Let $\phi: Q \rightarrow G$ be a representation and let $\alpha: Q \times G \rightarrow Q$, $(a, g) \mapsto a^{g}$, be a group action. We call the pair $(\phi, \alpha)$ an augmentation if $a * b=$ $a^{\phi(b)}$ and $\phi\left(a^{g}\right)=\phi(a)^{g}$ for all $a, b \in Q$ and $g \in G$. In other words, the following diagram commutes:

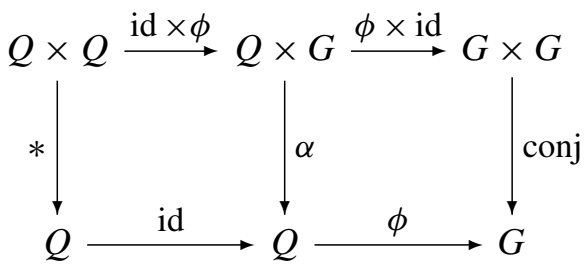


Remark 3.11. We will usually reinterpret the group action $\alpha$ as a group homomorphism $\bar{\alpha}: G \rightarrow \operatorname{Aut}(Q)$, and denote the augmentation by $Q \stackrel{\phi}{\longrightarrow} G \stackrel{\bar{\alpha}}{\longrightarrow} \operatorname{Aut}(Q)$. If $G$ is generated by the image $\phi(Q)$, then $\phi$ is equivariant and the action of $G$ on $Q$ is uniquely determined by the representation $\phi$. In this case we simply say that $\phi: Q \rightarrow G$ is an augmentation. For example, every quandle $Q$ comes equipped with the inner augmentation $\varrho: Q \rightarrow \operatorname{Inn}(Q)$.

Suppose that $Q$ is a quandle and $\phi: Q \rightarrow G$ is a representation on some group $G$. Obviously every quandle colouring $\tilde{f}: D \rightarrow Q$ maps to a group colouring $f=\phi \tilde{f}: D \rightarrow G$. If $\phi$ is an augmentation, then this process can be reversed, and we can replace quandle colourings by group colourings without any loss of information:

Lemma 3.12. Let $(Q, q) \stackrel{\phi}{\longrightarrow}(G, g) \stackrel{\bar{\alpha}}{\longrightarrow} \operatorname{Aut}(Q)$ be an augmentation of the quandle $Q$ with basepoint $q \in Q$ on the group $G$ with basepoint $x=\phi(q) \in G$. If $D$ is a long knot diagram, then every group colouring $f:(D, 0) \rightarrow(G, x)$ can be lifted to a unique quandle colouring $\tilde{f}:(D, 0) \rightarrow(Q, q)$ such that $f=\phi \tilde{f}$. In other words, $\phi$ induces a bijection

$$
\phi_{*}: \operatorname{Col}(D, 0 ; Q, q) \stackrel{\sim}{\longrightarrow} \operatorname{Col}(D, 0 ; G, x), \quad \tilde{f} \mapsto f=\phi \tilde{f} .
$$

The lifted colouring $\tilde{f}$ begins with $\tilde{f}(0)=q$ and ends with $\tilde{f}(n)=q^{\rho\left(l_{K}\right)}$, where $\rho:\left(\pi_{K}, m_{K}\right) \rightarrow(G, x)$ is the knot group representation associated with $f$.

Proof. Every representation $\phi:(Q, q) \rightarrow(G, x)$ induces a map $\phi_{*}$ sending each quandle colouring $\tilde{f}:(D, 0) \rightarrow(Q, q)$ to the associated group colouring $\phi \tilde{f}:$ $(D, 0) \rightarrow(G, x)$. In general $\phi_{*}$ is neither injective nor surjective, lest $\phi$ is an augmentation. To define the inverse map $\psi_{*}: \operatorname{Col}(D, 0 ; G, x) \rightarrow \operatorname{Col}(D, 0 ; Q, q)$, we use the action $\alpha: Q \times G \rightarrow Q$, which we temporarily denote by $(a, g) \mapsto a \bullet g$ for better readability.

The crucial ingredient in the proof is the commutativity of the bottom diagram on page 321. We first show how the condition $a * b=a \bullet \phi(b)$ ensures injectivity of $\phi_{*}$. Let $D$ be a long knot diagram with Wirtinger code $(\kappa, \varepsilon)$. Assume that $\tilde{f}, \hat{f}:(D, 0) \rightarrow(Q, q)$ are colourings with $\phi \tilde{f}=\phi \hat{f}$. By hypothesis we have $\tilde{f}(0)=\hat{f}(0)=q$. By induction suppose that $\tilde{f}(i-1)=\hat{f}(i-1)$ for some $i \geq 1$. In the case of a positive crossing $(\varepsilon i=+1)$ we then obtain

$$
\begin{aligned}
\tilde{f}(i) & =\tilde{f}(i-1) * \tilde{f}(\kappa i)=\tilde{f}(i-1) \bullet \phi \tilde{f}(\kappa i) \\
& =\hat{f}(i-1) \bullet \phi \hat{f}(\kappa i)=\hat{f}(i-1) * \hat{f}(\kappa i)=\hat{f}(i) .
\end{aligned}
$$

The case of a negative crossing $(\varepsilon i=-1)$ is analogous. We conclude that $\tilde{f}=\hat{f}$.

We now show how the equivariance condition $\phi(a \bullet g)=\phi(a) * g$ of the bottom diagram on page 321 ensures surjectivity. For every colouring $f:(D, 0) \rightarrow(G, x)$, 
denoted by $i \mapsto x_{i}$, the colours $x_{0}, \ldots, x_{n}$ satisfy $x_{i}=x_{i-1} * x_{\kappa i}^{\varepsilon i}$. We define partial longitudes $\ell_{0}, \ldots, \ell_{n}$ by setting $\ell_{i}:=\prod_{j=1}^{i} x_{j-1}^{-\varepsilon j} x_{\kappa j}^{\varepsilon j}$. In particular we have $x_{0}=x_{n}=x$ and $x_{i}=x_{0} * \ell_{i}$ for all $i=0, \ldots, n$. By definition, $\ell_{n}=\rho\left(l_{K}\right)$ is the (total) longitude of the colouring $f$. We define $\tilde{f}:(D, 0) \rightarrow(Q, q)$ by assigning the colour $q_{i}=q \bullet \ell_{i}$ to arc number $i=0, \ldots, n$. By hypothesis, $\phi: Q \rightarrow G$ is an equivariant map, whence

$$
\phi\left(q_{i}\right)=\phi\left(q \bullet \ell_{i}\right)=\phi(q) * \ell_{i}=x * \ell_{i}=x_{i} .
$$

At each positive crossing we find the following identity, using axiom (Q1):

$$
q_{i-1} * q_{\kappa i}=\left(q_{i-1} \bar{*} q_{i-1}\right) * q_{\kappa i}=\left(\left(\left(q \bullet \ell_{i-1}\right) \bullet x_{i-1}^{-1}\right) \cdot x_{\kappa i}=q \bullet \ell_{i}=q_{i} .\right.
$$

Analogously at each negative crossing:

$$
q_{i-1} \bar{*} q_{\kappa i}=\left(q_{i-1} * q_{i-1}\right) \bar{*} q_{\kappa i}=\left(\left(\left(q \bullet \ell_{i-1}\right) \bullet x_{i-1}\right) \cdot x_{\kappa i}^{-1}\right)=q \bullet \ell_{i}=q_{i} .
$$

We can thus define $\psi_{*}: \operatorname{Col}(D, 0 ; G, x) \rightarrow \operatorname{Col}(D, 0 ; Q, q)$ by $f \mapsto \tilde{f}$. Equation (1) shows that $\phi_{*} \psi_{*}=\mathrm{id}$, while (2) and (3) imply that $\psi_{*} \phi_{*}=$ id.

Remark 3.13. Obviously, the condition $a * b=a^{\phi(b)}$ cannot be dropped because it connects the quandle operation $*$ with the group action $\alpha$. Likewise, the equivariance condition $\phi\left(a^{g}\right)=\phi(a)^{g}$ cannot be dropped: as an extreme counterexample, consider a trivial quandle $Q=\{q\}$ and an arbitrary group $(G, x)$. We have a unique representation $\phi:(Q, q) \rightarrow(G, x)$ and a unique group action $\alpha: Q \times G \rightarrow Q$. The map $\phi$ is equivariant if and only if $x \in Z(G)$. In general $\phi_{*}$ cannot be a bijection, because the only $(Q, q)$-colouring is the trivial one, while there may be nontrivial $(G, x)$-colourings.

The Lifting Lemma has the following analogue for closed knots:

Lemma 3.14. Let $\phi:(Q, q) \rightarrow(G, x)$ be an augmentation of the quandle $Q$ on the group $G$. If $D$ is a closed knot diagram, then $\phi$ induces a bijection between $\operatorname{Col}(D, 0 ; Q, q)$ and those homomorphisms $\rho:\left(\pi_{K}, m_{K}\right) \rightarrow(G, x)$ satisfying $q^{\rho\left(l_{K}\right)}=q$.

As an immediate consequence we obtain:

Theorem 3.15. Every quandle colouring number $F_{Q}^{q}$ is the specialization of some knot colouring polynomial $P_{G}^{x}$.

Proof. We consider an augmentation $\phi:(Q, q) \rightarrow(G, x)$ with $G=\langle\phi(Q)\rangle$, for example the inner augmentation on $\phi: Q \rightarrow G=\operatorname{Inn}(Q)$ with basepoint $x=\phi(q)$.

For long knots, Lemma 3.12 implies $F_{Q}^{q}=F_{G}^{x}$. Hence $F_{Q}^{q}=\varepsilon P_{G}^{x}$, where $\varepsilon$ : $\mathbb{Z} G \rightarrow \mathbb{Z}$ is the augmentation map of the group ring, with $\varepsilon(g)=1$ for all $g \in G$.

For closed knots we define the linear map $\varepsilon: \mathbb{Z} G \rightarrow \mathbb{Z}$ by setting $\varepsilon(g)=1$ if $q^{g}=q$, and $\varepsilon(g)=0$ if $q^{g} \neq q$. Then Lemma 3.14 implies that $F_{Q}^{q}=\varepsilon P_{G}^{x}$. 
This argument will be generalized in Section 3E, where we show that every quandle 2-cocycle state-sum invariant is the specialization of some colouring polynomial.

3C. Quandle coverings, extensions, and cohomology. We recall how quandle colourings can be used to encode longitudinal information (see [Eisermann 2003] for details). We consider a long knot diagram with meridians $x_{0}, \ldots, x_{n}$ and partial longitudes $l_{0}, \ldots, l_{n}$ as defined in the above proof of the Lifting Lemma. In particular we have $x_{0}=x_{n}=m_{K}$ and $x_{i}=x_{0} * l_{i}$ with $l_{0}=1$ and $l_{n}=l_{K}$. If we colour each arc not only with its meridian $x_{i}$ but with the pair $\left(x_{i}, l_{i}\right)$, then at each crossing we find that

$$
x_{i}=x_{i-1} * x_{\kappa i}^{\varepsilon i} \quad \text { and } \quad l_{i}=l_{i-1} x_{i-1}^{-\varepsilon i} x_{\kappa i}^{\varepsilon i} .
$$

This crossing relation can be encoded in a quandle as follows.

Lemma 3.16 [Eisermann 2003]. Let $G$ be a group that is generated by a conjugacy class $Q=x^{G}$. Then $Q$ is a connected quandle with respect to conjugation $a * b=$ $b^{-1} a b$ and its inverse $a \bar{*} b=b a b^{-1}$. Let $G^{\prime}$ be the commutator subgroup and define

$$
\tilde{Q}=\tilde{Q}(G, x):=\left\{(a, g) \in G \times G^{\prime} \mid a=x^{g}\right\} .
$$

The set $\tilde{Q}$ becomes a connected quandle when equipped with the operations

$$
(a, g) *(b, h)=\left(a * b, g a^{-1} b\right) \quad \text { and }(a, g) \bar{*}(b, h)=\left(a \bar{*} b, g a b^{-1}\right) .
$$

The projection $p: \tilde{Q} \rightarrow Q$ given by $p(a, g)=a$ is a surjective quandle homomorphism. It becomes an equivariant map when we let $G^{\prime}$ act on $Q$ by conjugation and on $\tilde{Q}$ by $(a, g)^{b}=\left(a^{b}, g b\right)$. In both cases $G^{\prime}$ acts transitively and as a group of inner automorphisms.

The construction of the quandle $\tilde{Q}(G, x)$ has been tailor-made to capture longitude information. Considered purely algebraically, it is a covering in the following sense:

Definition 3.17. A surjective quandle homomorphism $p: \tilde{Q} \rightarrow Q$ is called a covering if $p(\tilde{x})=p(\tilde{y})$ implies $\tilde{a} * \tilde{x}=\tilde{a} * \tilde{y}$ for all $\tilde{a}, \tilde{x}, \tilde{y} \in \tilde{Q}$. In other words, the inner representation $\tilde{Q} \rightarrow \operatorname{Inn}(\tilde{Q})$ factors through $p$. This property allows us to define an action of $Q$ on $\tilde{Q}$ by setting $\tilde{a} * x:=\tilde{a} * \tilde{x}$ with $\tilde{x} \in p^{-1}(x)$.

In the construction of Lemma 3.16, the projection $p: \tilde{Q} \rightarrow Q$ is a covering map. Moreover, covering transformations are given by the left action of $\Lambda=C(x) \cap G^{\prime}$ defined by $\lambda \cdot(a, g)=(a, \lambda g)$. This action satisfies the following axioms:

(E1) $(\lambda \tilde{x}) * \tilde{y}=\lambda(\tilde{x} * \tilde{y})$ and $\tilde{x} *(\lambda \tilde{y})=\tilde{x} * \tilde{y}$ for all $\tilde{x}, \tilde{y} \in \tilde{Q}$ and $\lambda \in \Lambda$.

(E2) $\Lambda$ acts freely and transitively on each fibre $p^{-1}(x)$. 
Axiom (E1) is equivalent to saying that $\Lambda$ acts by automorphisms and the left action of $\Lambda$ commutes with the right action of $\operatorname{Inn}(\tilde{Q})$. We denote such an action by $\Lambda \curvearrowright \tilde{Q}$. In this situation the quotient $Q:=\Lambda \backslash \tilde{Q}$ carries a unique quandle structure that turns the projection $p: \tilde{Q} \rightarrow Q$ into a quandle covering.

Definition 3.18. An extension $E: \Lambda \curvearrowright \tilde{Q} \rightarrow Q$ consists of a surjective quandle homomorphism $\tilde{Q} \rightarrow Q$ and a group action $\Lambda \curvearrowright \tilde{Q}$ satisfying axioms (E1) and (E2). We call $E$ a central extension if $\Lambda$ is abelian.

Quandle extensions are an analogue of group extensions, and central quandle extensions come as close as possible to imitating central group extensions. Analogous to the case of groups, central quandle extensions are classified by the second cohomology group $H^{2}(Q, \Lambda)$. More precisely:

Theorem 3.19 [Eisermann 2003]. Let $Q$ be a quandle, let $\Lambda$ be an abelian group, and let $\mathscr{E}(Q, \Lambda)$ be the set of equivalence classes of central extensions of $Q$ by $\Lambda$. Given a central extension $E: \Lambda \curvearrowright \tilde{Q} \rightarrow Q$, each section $s: Q \rightarrow \tilde{Q}$ defines a 2-cocycle $\lambda: Q \times Q \rightarrow \Lambda$. If $s^{\prime}$ is another section, then the associated 2-cocycle $\lambda^{\prime}$ differs from $\lambda$ by a 2-coboundary. The map $E \mapsto[\lambda]$ so constructed induces a natural bijection $\mathscr{E}(Q, \Lambda) \cong H^{2}(Q, \Lambda)$.

The relevant portion of the cochain complex $C^{1} \stackrel{\delta^{1}}{\longrightarrow} C^{2} \stackrel{\delta^{2}}{\longrightarrow} C^{3}$ is formed by $n$-cochains $\lambda: Q^{n} \rightarrow \Lambda$ satisfying $\lambda\left(a_{1}, \ldots, a_{n}\right)=0$ whenever $a_{i}=a_{i+1}$ for some index $i$, and the first two coboundary operators $\delta^{1}(\mu)(a, b)=\mu(a)-\mu\left(a^{b}\right)$ and $\delta^{2}(\lambda)(a, b, c)=\lambda(a, c)-\lambda(a, b)+\lambda\left(a^{c}, b^{c}\right)-\lambda\left(a^{b}, c\right)$. For details, see [Carter et al. 1999; 2003b; Eisermann 2003].

3D. From colouring polynomials to state-sum invariants. Let $D$ be a knot diagram and let $f$ be a colouring of $D$ with colours in $Q$. Suppose that $\Lambda$ is an abelian group, written multiplicatively, and that $\lambda: Q^{2} \rightarrow \Lambda$ is a 2-cocycle. For each coloured crossing $p$ as in Figure 4 , we define its weight by $\langle\lambda \mid p\rangle:=\lambda(a, b)^{\varepsilon}$. The total weight of the colouring $f$ is the product $\langle\lambda \mid f\rangle:=\prod_{p}\langle\lambda \mid p\rangle$ over all crossings $p$. The state-sum of the diagram $D$ is defined to be $S_{Q}^{\lambda}(D):=\sum_{f}\langle\lambda \mid f\rangle$, where the sum in $\mathbb{Z} \Lambda$ is taken over all colourings $f: D \rightarrow Q$. We recall some results:

Lemma 3.20 [Carter et al. 1999; 2003b]. The state-sum $S_{Q}^{\lambda}$ is invariant under Reidemeister moves and thus defines a knot invariant $S_{Q}^{\lambda}: \mathscr{K} \rightarrow \mathbb{Z} \Lambda$.

Lemma 3.21 [Carter et al. 2003b, Prop.4.5]. If the colouring $f: D \rightarrow Q$ is closed, that is $f(0)=f(n)$, then the weight $\langle\lambda \mid f\rangle$ is invariant under addition of coboundaries. As a consequence, the state sum $S_{Q}^{\lambda}$ of a closed knot depends only on the cohomology class $[\lambda]$. 
Lemma 3.22 [Eisermann 2005, Lem. 32]. The diagonal action of $\operatorname{Inn}(Q)$ on $Q^{n}$ induces the trivial action on $H^{*}(Q, \Lambda)$. As a consequence, for each closed colouring $f: D \rightarrow Q$ and every inner automorphism $g \in \operatorname{Inn}(Q)$ we have $\left\langle\lambda \mid f^{g}\right\rangle=$ $\left\langle{ }^{g} \lambda \mid f\right\rangle=\langle\lambda \mid f\rangle$.

This last result is well-known in group cohomology [Brown 1982, Prop. II.6.2]. It seems to be folklore in quandle cohomology, but I could not find a written account of it. The necessary argument is provided by [Eisermann 2005, Lem. 32] in the more general setting of Yang-Baxter cohomology, which immediately translates to Lemma 3.22.

Lemma 3.23 [Eisermann 2003, Lem. 50]. Let $p:(\tilde{Q}, \tilde{q}) \rightarrow(Q, q)$ be a central quandle extension. Given a long knot diagram $D$, every colouring $f:(D, 0) \rightarrow$ $(Q, q)$ uniquely lifts to a colouring $\tilde{f}:(D, 0) \rightarrow(\tilde{Q}, \tilde{q})$ such that $f=p \tilde{f}$. If $f$ is closed then $\tilde{f}(n)=\langle\lambda \mid f\rangle \cdot \tilde{q}$, where $[\lambda] \in H^{2}(Q, \Lambda)$ is the cohomology class associated with the extension $p$.

These preliminaries being in place, we can now prove that every colouring polynomial $P_{G}^{x}$ can be presented as a 2-cocycle state-sum invariant, provided that the subgroup $\Lambda=C(x) \cap G^{\prime}$ is abelian.

Theorem 3.24. Suppose that $G$ is a colouring group with basepoint $x$ such that the subgroup $\Lambda=C(x) \cap G^{\prime}$ is abelian. Then the colouring polynomial $P_{G}^{x}$ can be presented as a quandle 2-cocycle state-sum invariant. More precisely, the quandle $Q=x^{G}$ admits a 2-cocycle $\lambda \in Z^{2}(Q, \Lambda)$ such that $S_{Q}^{\lambda}=P_{G}^{x} \cdot|Q|$.

Proof. Let $Q=x^{G}$ be the conjugacy class of $x$ in the group $G$, and let $\tilde{Q}=\tilde{Q}(G, x)$ be the covering quandle constructed in Lemma 3.16. Since $\Lambda$ is abelian, we obtain a central extension $\Lambda \curvearrowright \tilde{Q} \rightarrow Q$. Let $[\lambda] \in H^{2}(Q, \Lambda)$ be the associated cohomology class. As basepoints we choose $q=x$ in $Q$ and $\tilde{q}=(x, 1)$ in $\tilde{Q}$.

Let $D$ be a long diagram of some knot $K$, let $f:(D, 0) \rightarrow(Q, q)$ be a colouring, let $\rho:\left(\pi_{K}, m_{K}\right) \rightarrow(G, x)$ be the corresponding knot group homomorphism, and let $\tilde{f}:(D, 0) \rightarrow(\tilde{Q}, \tilde{q})$ be the lifting of $f$. On the one hand we have $\tilde{f}(n)=$ $(x,\langle\lambda \mid f\rangle)$ from Lemma 3.23. On the other hand we have $\tilde{f}(n)=\left(x, \rho\left(l_{K}\right)\right)$ from the Wirtinger presentation. Thus $\rho\left(l_{K}\right)=\langle\lambda \mid f\rangle$, and summing over all colourings $f:(D, 0) \rightarrow(Q, q)$ yields $P_{G}^{x}(K)$.

To obtain the state-sum $S_{Q}^{\lambda}$ we have to sum over all colourings $f: D \rightarrow Q$. We have $\operatorname{Col}(D, Q)=\cup_{q^{\prime} \in Q} \operatorname{Col}\left(D, 0 ; Q, q^{\prime}\right)$. Since $Q$ is connected, for each $q^{\prime} \in Q$ there exists $g \in G$ such that $q^{g}=q^{\prime}$. Hence $f \mapsto f^{g}$ establishes a bijection between $\operatorname{Col}(D, 0 ; Q, q)$ and $\operatorname{Col}\left(D, 0 ; Q, q^{\prime}\right)$. By Lemma 3.22 we have $\langle\lambda \mid f\rangle=\left\langle\lambda \mid f^{g}\right\rangle$. Thus the state-sum over all colourings $f:(D, 0) \rightarrow\left(Q, q^{\prime}\right)$ again yields $P_{G}^{x}$. We conclude that $S_{Q}^{\lambda}(K)=P_{G}^{x}(K) \cdot|Q|$. 
3E. From state-sum invariants to colouring polynomials. Theorem 3.24 has the following converse, which allows us to express quandle 2-cocycle state-sum invariants by knot colouring polynomials.

Theorem 3.25. Every quandle 2-cocycle state-sum invariant of knots is the specialization of some knot colouring polynomial. More precisely, suppose that $Q$ is a connected quandle, $\Lambda$ is an abelian group, and $\lambda \in Z^{2}(Q, \Lambda)$ is a 2-cocycle with associated invariant $S_{Q}^{\lambda}: \mathscr{K} \rightarrow \mathbb{Z} \Lambda$. Then there exists a group $G$ with basepoint $x$ and a linear map $\varphi: \mathbb{Z} G \rightarrow \mathbb{Z} \Lambda$ such that the colouring polynomial $P_{G}^{x}: \mathscr{K} \rightarrow \mathbb{Z} G$ satisfies $S_{Q}^{\lambda}=\varphi P_{G}^{x} \cdot|Q|$.

Proof. We first construct a suitable group $(G, x)$ together with a linear map $\varphi$ : $\mathbb{Z} G \rightarrow \mathbb{Z} \Lambda$. Let $\Lambda \curvearrowright \tilde{Q} \stackrel{p}{\longrightarrow} Q$ be the central extension associated with the 2-cocycle $\lambda$, as explained in Theorem 3.19. We put $G:=\operatorname{Inn}(\tilde{Q})$. The inner representation $\tilde{\varrho}: \tilde{Q} \rightarrow G$ defines an augmented quandle in the sense of Section 3B. We choose a basepoint $\tilde{q} \in \tilde{Q}$ and set $x:=\tilde{\varrho}(\tilde{q})$.

We choose $q=p(\tilde{q})$ as basepoint of $Q$. Let $s: Q \rightarrow \tilde{Q}$ be a section that realizes the 2-cocycle $\lambda$. Since $p$ is a covering, we obtain a representation $\varrho: Q \rightarrow G$ by $\varrho=\tilde{\varrho} \circ s$. Conversely, we can define an action of $G$ on $Q$ by setting $a^{g}=p\left(s(a)^{g}\right)$. This turns the representation $\varrho: Q \rightarrow G$ into an augmentation and $p: \tilde{Q} \rightarrow Q$ into an equivariant map. Our notation being in place, we can now define the linear map $\varphi: \mathbb{Z} G \rightarrow \mathbb{Z} \Lambda$ by setting $\varphi(g)= \begin{cases}0 & \text { if } q^{g} \neq q, \\ \ell & \text { if } q^{g}=q \text { and } \ell \in \Lambda \text { such that } \tilde{q}^{g}=\ell \cdot \tilde{q} .\end{cases}$

It remains to prove that $S_{Q}^{\lambda}=\varphi P_{G}^{x} \cdot|Q|$. Let $K$ be a knot represented by a long knot diagram $D$. The Lifting Lemma 3.14 grants us a bijection between closed colourings $f:(D, 0) \rightarrow(Q, q)$ and those homomorphisms $\rho:\left(\pi_{K}, m_{K}\right) \rightarrow(G, x)$ that satisfy $q^{\rho\left(l_{K}\right)}=q$. Regarding the covering $\tilde{Q}$, we claim that $\tilde{q}^{\rho\left(l_{K}\right)}=\langle\lambda \mid f\rangle \cdot \tilde{q}$. To see this, let $\tilde{f}:(D, 0) \rightarrow(\tilde{Q}, \tilde{q})$ be the lifting of $f$. On the one hand we can apply the Lifting Lemma 3.14 to the augmentation $\tilde{Q} \rightarrow G$, which yields $\tilde{f}(n)=\tilde{q}^{\rho\left(l_{K}\right)}$. On the other hand we can apply Lemma 3.23, which yields $\tilde{f}(n)=\langle\lambda \mid f\rangle \cdot \tilde{q}$.

The map $\varphi$ thus specializes the knot colouring polynomial $P_{G}^{x}(K)$ to the statesum $\sum_{f}\langle\lambda \mid f\rangle$, at least if we restrict the summation to colourings $f:(D, 0) \rightarrow$ $(Q, q)$. Since $Q$ is connected, any other basepoint $q^{\prime}$ yields the same state-sum by Lemma 3.22. Summing over all $q^{\prime} \in Q$, we thus obtain $S_{Q}^{\lambda}=\varphi P_{G}^{x} \cdot|Q|$, as claimed.

\section{Colouring polynomials are Yang-Baxter invariants}

Freyd and Yetter [1989] have shown that the colouring number $F_{G}^{x}: \mathscr{K} \rightarrow \mathbb{Z}$ is a Yang-Baxter invariant. This means that $F_{G}^{x}$ can be obtained as the trace of a linear braid group representation arising from a suitable Yang-Baxter operator $c$. 
In this section we will show that the colouring polynomial $P_{G}^{x}: \mathscr{K} \rightarrow \mathbb{Z} \Lambda$ is also a Yang-Baxter invariant, obtained from a certain Yang-Baxter operator $\tilde{c}$ defined below. It will follow from our construction that $\tilde{c}$ is a deformation of $c$ over $\mathbb{Z} \Lambda$.

4A. Braid group representations and Yang-Baxter invariants. The existence of Yang-Baxter invariants rests on two classical theorems: Artin's presentation of the braid groups and the Alexander-Markov theorem, which we will now recall. Our exposition closely follows [Eisermann 2005] and is included here for convenience.

Theorem 4.1 [Artin 1947]. The braid group on $n$ strands can be presented as

$$
\mathrm{B}_{n}=\left\langle\begin{array}{lll}
\sigma_{1}, \ldots, \sigma_{n-1} \mid \begin{array}{cc}
\sigma_{i} \sigma_{j}=\sigma_{j} \sigma_{i} & \text { for }|i-j| \geq 2 \\
\sigma_{i} \sigma_{j} \sigma_{i}=\sigma_{j} \sigma_{i} \sigma_{j} & \text { for }|i-j|=1
\end{array}
\end{array}\right\},
$$

where the braid $\sigma_{i}$ performs a positive half-twist of the strands $i$ and $i+1$.

Definition 4.2. Let $\mathbb{K}$ be a commutative ring and $V$ a $\mathbb{K}$-module. A Yang-Baxter operator (or R-matrix) is an automorphism $c: V \otimes V \rightarrow V \otimes V$ that satisfies the Yang-Baxter equation, also called braid relation:

$$
\left(c \otimes \mathrm{id}_{V}\right)\left(\mathrm{id}_{V} \otimes c\right)\left(c \otimes \mathrm{id}_{V}\right)=\left(\operatorname{id}_{V} \otimes c\right)\left(c \otimes \mathrm{id}_{V}\right)\left(\operatorname{id}_{V} \otimes c\right) \quad \text { in } \operatorname{Aut}_{\mathbb{}}\left(V^{\otimes 3}\right) .
$$

Here and in the sequel tensor products are taken over $\mathbb{K}$ if no other ring is indicated.

Corollary 4.3. Given a Yang-Baxter operator $c$ and some integer $n \geq 2$, we can define automorphisms $c_{i}: V^{\otimes n} \rightarrow V^{\otimes n}$ by setting

$$
c_{i}=\mathrm{id}_{V}^{\otimes(i-1)} \otimes c \otimes \mathrm{id}_{V}^{\otimes(n-i-1)} \quad \text { for } i=1, \ldots, n-1 .
$$

The Artin presentation implies that there exists, for each $n$, a unique braid group representation $\rho_{c}^{n}: \mathrm{B}_{n} \rightarrow \operatorname{Aut}_{\mathbb{K}}\left(V^{\otimes n}\right)$ defined by $\rho_{c}^{n}\left(\sigma_{i}\right)=c_{i}$.

We orient braids from right to left as in Figure 6. Braid groups will act on the left, so that composition of braids corresponds to the usual composition of maps. The passage from braids to links is granted by the closure map []: $\bigcup_{n} \mathrm{~B}_{n} \rightarrow \mathscr{L}$ defined as follows: for each braid $\beta$ we define its closure $[\beta]$ to be the link in $\mathbb{S}^{3}$ obtained by identifying opposite endpoints, as indicated in Figure 6.
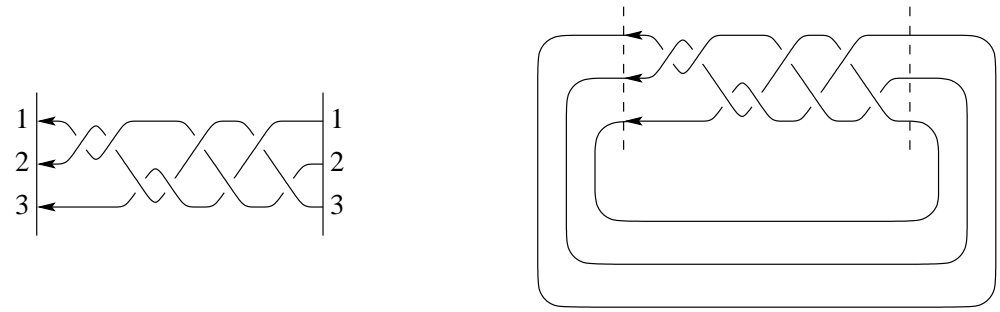

Figure 6. A braid $\beta$ and its closure $[\beta]$. 
Theorem 4.4 (Alexander and Markov; see [Birman 1974]). Every link can be represented as the closure of some braid. Two braids represent the same link if and only if one can be transformed into the other by a finite sequence of the following Markov moves:

(M1) Pass from $\beta \in \mathrm{B}_{n}$ to $\beta \sigma_{n}^{ \pm 1} \in \mathrm{B}_{n+1}$, or vice versa.

(stabilization)

(M2) Pass from $\beta \in \mathrm{B}_{n}$ to $\alpha^{-1} \beta \alpha$ with $\alpha \in \mathrm{B}_{n}$.

(conjugation)

Constructing a link invariant $F: \mathscr{L} \rightarrow \mathbb{K}$ is thus equivalent to constructing a map $F: \bigcup_{n} \mathrm{~B}_{n} \rightarrow \mathbb{K}$ that is invariant under Markov moves. The most natural approach is to consider traces of linear braid group representations: invariance under conjugation is automatic, so we only have to require invariance under stabilization:

Definition 4.5. Suppose that $V$ is a free $\mathbb{K}$-module with finite basis. Let $c: V \otimes V \rightarrow$ $V \otimes V$ be a Yang-Baxter operator. An automorphism $m: V \rightarrow V$ is called Markov operator for $c$ if it satisfies
$(\mathrm{m} 1) \operatorname{tr}_{2}\left((m \otimes m) \circ c^{ \pm 1}\right)=m$,
(the trace condition)
$(\mathrm{m} 2) c \circ(m \otimes m)=(m \otimes m) \circ c$.
(commutativity)

Here the partial trace $\operatorname{tr}_{2}: \operatorname{End}(V \otimes V) \rightarrow \operatorname{End}(V)$ is defined as follows. Let $\left(v_{1}, \ldots, v_{n}\right)$ be a basis of $V$ over $\mathbb{K}$. Every $f \in \operatorname{End}(V \otimes V)$ uniquely corresponds to a matrix $f_{i j}^{k \ell}$ such that $f\left(v_{i} \otimes v_{j}\right)=\sum_{k, \ell} f_{i j}^{k \ell} v_{k} \otimes v_{\ell}$. We can then define $g=\operatorname{tr}_{2}(f) \in \operatorname{End}(V), g\left(v_{i}\right)=\sum_{k} g_{i}^{k} v_{k}$, by the matrix $g_{i}^{k}=\sum_{j} f_{i j}^{k j}$. See [Kassel 1995 , §II.3].

Corollary 4.6. Given a Yang-Baxter operator $c$ with Markov operator $m$, we define a family of maps $F_{n}: \mathrm{B}_{n} \rightarrow \mathbb{K}$ by $F_{n}(\beta)=\operatorname{tr}\left(m^{\otimes n} \circ \rho_{c}^{n}(\beta)\right)$. Then the induced map $F: \bigcup_{n} \mathrm{~B}_{n} \rightarrow \mathbb{K}$ is invariant under both Markov moves and thus defines a link invariant $F: \mathscr{L} \rightarrow \mathbb{K}$.

The proof of this corollary is straight-forward: the trace condition (m1) implies invariance under stabilization (M1), and commutativity (m2) implies invariance under conjugation (M2). Much more intricate is the question how to actually find such a Yang-Baxter-Markov operator $(c, m)$. Attempts to construct solutions in a systematic way have led to the theory of quantum groups [Drinfel'd 1987]. For details we refer to the concise introduction [Kassel et al. 1997] or the textbook [Kassel 1995].

Remark 4.7. For some Yang-Baxter operators $c$ there does not exist any Markov operator $m$ at all. If it exists, $m$ is in general not the identity, as in the case of the Jones polynomial or other quantum invariants. The Yang-Baxter operators derived from knot diagram colourings below are very special in that they allow the Markov operator $m=\mathrm{id}$, which is equivalent to saying that $\operatorname{tr}_{2}\left(c^{ \pm 1}\right)=\mathrm{id}$. 
4B. Colouring polynomials of long knots. Before we consider colouring polynomials, let us first recall how colouring numbers can be obtained from a suitable Yang-Baxter operator.

Theorem 4.8 [Freyd and Yetter 1989, Prop. 4.2.5 and the remark following its proof]. Let $Q$ be a quandle and let $\mathbb{K} Q$ be the free $\mathbb{K}$-module with basis $Q$. The quandle structure of $Q$ can be linearly extended to a Yang-Baxter operator

$$
c_{Q}: \mathbb{K} Q \otimes \mathbb{K} Q \rightarrow \mathbb{K} Q \otimes \mathbb{K} Q \quad \text { with } a \otimes b \mapsto b \otimes(a * b) \quad \text { for all } a, b \in Q \text {. }
$$

Axiom $(Q 2)$ ensures that $c_{Q}$ is an automorphism, while Axiom (Q3) implies the Yang-Baxter equation. If $Q$ is finite, then $(Q 1)$ ensures that $\operatorname{tr}_{2}\left(c_{Q}^{ \pm 1}\right)=\mathrm{id}$. In this case the corresponding Yang-Baxter invariant $F_{Q}=\operatorname{tr} \circ \rho_{Q}$ coincides with the number of $Q$-colourings (defined in Section 3A) followed by the ring homomorphism $\mathbb{Z} \rightarrow \mathbb{K}$.

As an example consider a finite group $G$ with basepoint $x$. The Yang-Baxter operator constructed from the quandle $Q=x^{G}$ then leads to the colouring number $F_{Q}=F_{G}^{x} \cdot|Q|$.

We will now move from colouring numbers to colouring polynomials: consider the quandle extension $\Lambda \curvearrowright \tilde{Q} \rightarrow Q$ as defined in Section 3C, where the quandle $Q=Q(G, x)$ is covered by $\tilde{Q}=\tilde{Q}(G, x)$, and the deck transformation group is $\Lambda=C(x) \cap G^{\prime}$. As before, we linearly extend the quandle structure of $\tilde{Q}$ to a YangBaxter operator $c_{\tilde{Q}}$, and denote the associated linear braid group representation by $\rho_{\tilde{Q}}$. We will, however, not take the total trace as before, but rather use the partial trace $\operatorname{tr}^{\prime}: \operatorname{End}_{\mathbb{K}}\left(\mathbb{K} \tilde{Q}^{\otimes n}\right) \rightarrow \operatorname{End}_{\mathbb{}}(\mathbb{K} \tilde{Q})$, contracting the tensor factors $2, \ldots, n$.

Theorem 4.9. Let $(G, x)$ be a finite group such that the conjugacy class $Q=$ $x^{G}$ generates $G$. Let $\tilde{Q}=\tilde{Q}(G, x)$ be the covering quandle and let $\rho_{\tilde{Q}}$ be the associated braid group representation. Suppose that the knot $K$ is represented by a braid $\beta$. Then the partial trace $\operatorname{tr}^{\prime}\left(\rho_{\tilde{Q}}(\beta)\right): \mathbb{K} \tilde{Q} \rightarrow \mathbb{K} \tilde{Q}$ is given by multiplication with $P_{G}^{x}(K)$.

Note that the free left action of $\Lambda$ on $\tilde{Q}$ turns $\mathbb{K} \tilde{Q}$ into a free left module over $\mathbb{K} \Lambda$. In particular, multiplication by $P_{G}^{x}(K)$ is a $\mathbb{K}$-linear endomorphism. If $\mathbb{K}$ is of characteristic 0 , then the endomorphism $\operatorname{tr}^{\prime}\left(\rho_{\tilde{Q}}(\beta)\right)$ uniquely determines $P_{G}^{x}(K)$.

Proof. We use the obvious bases $\tilde{Q}$ for $\mathbb{R} \tilde{Q}$ and $\tilde{Q}^{n}$ for $\mathbb{K} \tilde{Q}^{\otimes n}$. Each endomorphism $f: \mathbb{K} \tilde{Q}^{\otimes n} \rightarrow \mathbb{K} \tilde{Q}^{\otimes n}$ is then represented by a matrix $M_{q_{1} q_{2} \ldots q_{n}}^{p_{1} p_{2} \ldots p_{n}}$, indexed by elements $p_{i}$ and $q_{j}$ in the basis $\tilde{Q}$. The partial $\operatorname{trace} \operatorname{tr}^{\prime}(f): \mathbb{K} \tilde{Q} \rightarrow \mathbb{R} \tilde{Q}$ is given by the matrix $T_{q_{1}}^{p_{1}}=\sum M_{q_{1} p_{2} \ldots p_{n}}^{p_{1} p_{2} \ldots p_{n}}$, where the sum is taken over all repeated indices $p_{2}, \ldots, p_{n}$.

By construction, each elementary braid $\sigma_{i}$ acts as a permutation on the basis $\tilde{Q}^{n}$, thus each braid $\beta \in \mathrm{B}_{n}$ is represented by a permutation matrix with respect to 
this basis. We interpret this action as colouring the braid $\beta$ with elements of $\tilde{Q}$ : we colour the right ends of the braid with $v=p_{1} \otimes \cdots \otimes p_{n}$. Moving from right to left, at each crossing the new arc is coloured according to the Wirtinger rule as depicted in Figure 4. We thus arrive at the left ends of the braid being coloured with $\rho(\beta) v=q_{1} \otimes \cdots \otimes q_{n}$. We conclude that colourings of the braid $\beta$ that satisfy the trace conditions $p_{2}=q_{2}, \ldots, p_{n}=q_{n}$ are in natural bijection with colourings of the corresponding long knot $K$.

We now turn to the remaining indices $p_{1}$ and $q_{1}$. Let us first consider the special case $p_{1}=(x, 1)$ and $q_{1}=(y, \lambda)$. From the preceding argument we see that $T_{q_{1}}^{p_{1}}$ equals the number of $\tilde{Q}$-colourings of the long knot $K$ that start with $(x, 1)$ and end with $(y, \lambda)$. According to Lemma 3.12, such colourings exist only for $y=x$ and $\lambda \in \Lambda$, hence we have $q_{1}=\lambda \cdot p_{1}$. We conclude that $T_{q_{1}}^{p_{1}}$ equals the number of representations $\left(\pi_{K}, m_{K}, l_{K}\right) \rightarrow(G, x, \lambda)$. In total we get

$$
\operatorname{tr}^{\prime}(\rho(\beta))\left(p_{1}\right)=P_{G}^{x}(K) \cdot p_{1} .
$$

The preceding construction is equivariant under the right-action of the group $G^{\prime}$ on the covering quandle $\tilde{Q}$. According to Lemma 3.16 this action is transitive: for every $p \in \tilde{Q}$ there exists $g \in G^{\prime}$ and $p=p_{1}^{g}$, so we conclude that

$$
\operatorname{tr}^{\prime}(\rho(\beta))(p)=P_{G}^{x}(K) \cdot p .
$$

This means that the endomorphism $\operatorname{tr}^{\prime}(\rho(\beta)): \mathbb{K} \tilde{Q} \rightarrow \mathbb{K} \tilde{Q}$ is given by multiplication with $P_{G}^{x}(K)$.

Remark 4.10. The partial trace $\operatorname{tr}^{\prime}: \operatorname{End}_{\mathbb{}}\left(\mathbb{K} \tilde{Q}^{\otimes n}\right) \rightarrow \operatorname{End}_{\mathbb{}}(\mathbb{R} \tilde{Q})$ corresponds to closing the strands $2, \ldots, n$ of the braid $\beta$, but leaving the first strand open: the object thus represented is a long knot. The natural setting for such constructions is the category of tangles and its linear representations [Kassel 1995]. The previous theorem then says that the long knot $K$ is represented by the endomorphism $\mathbb{K} \tilde{Q} \rightarrow$ $\mathbb{K} \tilde{Q}$ that is given by multiplication with $P_{G}^{x}(K)$.

If we used the complete trace tr $: \operatorname{End}_{\mathbb{K}}\left(\mathbb{K} \tilde{Q}^{\otimes n}\right) \rightarrow \mathbb{K}$ instead, then we would obtain a different invariant $F_{\tilde{Q}}=\operatorname{tr} \circ \rho_{\tilde{Q}}$. By the preceding arguments, $F_{\tilde{Q}}(K)$ equals $|\tilde{Q}|$ times the number of representations $\left(\pi_{K}, m_{K}, l_{K}\right) \rightarrow(G, x, 1)$, which corresponds to the coefficient of the unit element in the colouring polynomial $P_{G}^{x}(K)$.

4C. Colouring polynomials of closed knots. We now show how the colouring polynomial $P_{G}^{x}$ of closed knots can be obtained as the trace of a suitable YangBaxter representation. To this end we will modify the construction of the preceding paragraph in order to replace the partial trace $\operatorname{tr}^{\prime}$ by the complete trace tr. 
We proceed as follows: the quandle $Q=x^{G}$ admits an extension $\Lambda \curvearrowright \tilde{Q} \rightarrow Q$ as defined in Section 3C. The quandle structure of $\tilde{Q}$ linearly extends to a YangBaxter operator $c_{\tilde{Q}}$ on $\mathbb{K} \tilde{Q}$. The free $\Lambda$-action on $\tilde{Q}$ turns $\mathbb{K} \tilde{Q}$ into a free module over $\mathbb{A}=\mathbb{K} \Lambda$. If $\Lambda$ is abelian, we can pass to an $\mathbb{A}$-linear operator

$$
\tilde{c}_{Q}: \mathbb{K} \tilde{Q} \otimes_{\mathbb{A}} \mathbb{K} \tilde{Q} \rightarrow \mathbb{K} \tilde{Q} \otimes_{\mathbb{A}} \mathbb{K} \tilde{Q} \quad \text { with } \quad \tilde{a} \otimes \tilde{b} \mapsto \tilde{b} \otimes(\tilde{a} * \tilde{b}) \quad \text { for all } \quad \tilde{a}, \tilde{b} \in \tilde{Q} .
$$

The difference between $c_{\tilde{Q}}$ and $\tilde{c}_{Q}$ is that the tensor product is now taken over $\mathbb{A}$, which means that everything is bilinear with respect to multiplication by $\lambda \in \Lambda$. In the following theorem and its proof all tensor products are to be taken over the ring $\mathbb{A}$, but for notational simplicity we will write $\otimes$ for $\otimes_{\mathbb{A}}$.

Theorem 4.11. If $(G, x)$ is a colouring group such that $\Lambda=C(x) \cap G^{\prime}$ is abelian, then the colouring polynomial $P_{G}^{x}: \mathscr{K} \rightarrow \mathbb{Z} \Lambda$ is a Yang-Baxter invariant. More precisely, the preceding construction yields a Yang-Baxter-Markov operator ( $\tilde{c}_{Q}$, id) over the ring $\mathbb{A}=\mathbb{K} \Lambda$, and the associated knot invariant satisfies $\tilde{F}_{Q}=\varphi P_{G}^{x} \cdot|Q|$ where $\varphi: \mathbb{Z} \Lambda \rightarrow \mathbb{K} \Lambda$ is the natural ring homomorphism defined by $\varphi(\lambda)=\lambda$ for all $\lambda \in \Lambda$.

If $\mathbb{K}$ is of characteristic 0 , then $\tilde{F}_{Q}$ is equivalent to the knot colouring polynomial $P_{G}^{x}$. If $\mathbb{K}$ is of finite characteristic, then we may lose some information and $\tilde{F}_{Q}$ is usually weaker than $P_{G}^{x}$. In the worst case $|Q|$ vanishes in $\mathbb{K}$ and $\tilde{F}_{Q}$ becomes trivial.

Proof. It is a routine calculation to prove that $\tilde{c}_{Q}$ is a Yang-Baxter operator over A: as before, axiom $(\mathrm{Q} 2)$ implies that $\tilde{c}_{Q}$ is an automorphism, while axiom $(\mathrm{Q} 3)$ ensures that $\tilde{c}_{Q}$ satisfies the Yang-Baxter equation. Axiom (Q1) implies the trace condition $\operatorname{tr}_{2}\left(\tilde{c}_{Q}^{ \pm 1}\right)=\mathrm{id}$, hence $\left(\tilde{c}_{Q}, \mathrm{id}\right)$ is a Yang-Baxter-Markov operator. We thus obtain a linear braid group representation $\tilde{\rho}_{Q}^{n}: \mathrm{B}_{n} \rightarrow \operatorname{Aut}_{\mathbb{A}}\left(\mathbb{K} \tilde{Q}^{\otimes n}\right)$, whose character $\tilde{F}_{Q}=\operatorname{tr} \circ \tilde{\rho}_{Q}$ is Markov invariant and induces a link invariant $\tilde{F}_{Q}: \mathscr{L} \rightarrow$ A. Restricted to knots we claim that $\tilde{F}_{Q}=P_{G}^{x} \cdot|Q|$. The proof of the theorem parallels the proof of Theorem 4.9, but requires some extra care.

To represent $\tilde{c}_{Q}$ by a matrix, we have to choose a basis of $\mathbb{R} \tilde{Q}$ over $\mathbb{A}$. Let $s: Q \rightarrow \tilde{Q}$ be a section to the central extension $\Lambda \curvearrowright \tilde{Q} \rightarrow Q$. Then $B=s(Q)$ is a basis of $\mathbb{R} \tilde{Q}$ as an $\mathbb{A}$-module. For the basepoint $x$ we can assume $s(x)=(x, 1)$, but otherwise there are no canonical choices. In general, $s$ will not (and cannot) be a homomorphism of quandles, but we have $s(a) * s(b)=\lambda(a, b) \cdot s(a * b)$ with a certain 2-cocycle $\lambda: Q \times Q \rightarrow \Lambda$ that measures the deviation of $s$ from being a homomorphism. Just as $c_{Q}$ is represented by a permutation matrix, we see that $\tilde{c}_{Q}$ is represented by the same matrix except that the 1's are replaced with the elements $\lambda(a, b) \in \Lambda$. This is usually called a monomial matrix or generalized permutation matrix. 
Since $\mathbb{K} \tilde{Q}$ is a free $\mathbb{A}$-module with finite basis $B=s(Q)$, the tensor product $\mathbb{K} \tilde{Q}^{\otimes n}$ is also free and has finite basis $B^{n}$. The trace tro $\tilde{\rho}(\beta)$ is calculated as the sum $\sum_{v \in B^{n}}\langle\tilde{\rho}(\beta) v \mid v\rangle$. Note that $\tilde{\rho}(\beta)$ is again a monomial matrix in the sense that each row and each column has exactly one nonzero entry. Hence a vector $v \in B^{n}$ contributes to the trace sum if and only if $\tilde{\rho}(\beta) v=\lambda(v) v$ with some $\lambda(v) \in \Lambda$. It remains to characterize eigenvectors and identify their eigenvalues.

Given a braid $\beta \in \mathrm{B}_{n}$ we can interpret the action of $\tilde{\rho}(\beta)$ as colouring the braid $\beta$ : we colour the right ends of the braid with a basis vector $v \in B^{n}$,

$$
v=\left(a_{1}, g_{1}\right) \otimes\left(a_{2}, g_{2}\right) \otimes \ldots \otimes\left(a_{n}, g_{n}\right) .
$$

Moving from right to left, at each crossing the new arc is coloured according to the Wirtinger rule as depicted in Figure 4. We thus arrive at the left ends of the braid, being coloured with

$$
\tilde{\rho}(\beta) v=\left(b_{1}, h_{1}\right) \otimes\left(b_{2}, h_{2}\right) \otimes \cdots \otimes\left(b_{n}, h_{n}\right) .
$$

Since the tensor product is defined over $\mathbb{A}$, we have $\tilde{\rho}(\beta) v=\lambda(v) v$ if and only if $a_{1}=b_{1}, a_{2}=b_{2}, \ldots, a_{n}=b_{n}$. Hence each eigenvector $v \in B^{n}$ naturally corresponds to a $Q$-colouring of the closed braid $K=[\beta]$.

In order to identify the eigenvalue $\lambda(v)$, we will further assume that $\left(a_{1}, g_{1}\right)=$ $(x, 1)$, where $x$ is the basepoint of $G$. Such an eigenvector will be called normalized. Using the tensor product-structure over $\mathbb{A}=\mathbb{K} \Lambda$, we obtain

$$
\tilde{\rho}(\beta) v=(x, \lambda) \otimes\left(a_{2}, g_{2}\right) \otimes \cdots \otimes\left(a_{n}, g_{n}\right)=\lambda(v) v
$$

as in the proof of Theorem 4.9. We conclude that each normalized eigenvector $v \in B^{n}$ with $\tilde{\rho}(\beta) v=\lambda(v) v$ corresponds to a $\tilde{Q}$-colouring of the long knot, where the first arc is coloured by $(x, 1)$ and the last arc is coloured by $(x, \lambda)$. This means that the eigenvalue $\lambda(v)$ is the associated colouring longitude.

We finally show that $\tilde{F}_{Q}=P_{G}^{x} \cdot|Q|$ by calculating the trace $\sum_{v \in B^{n}}\langle\tilde{\rho}(\beta) v \mid v\rangle$. Normalized eigenvectors $v \in\{(x, 1)\} \times B^{n-1}$ with $\tilde{\rho}(\beta) v=\lambda(v) v$ correspond to colourings $\rho:\left(\pi_{K}, m_{k}\right) \rightarrow(G, x)$ with $\rho\left(l_{K}\right)=\lambda(v)$. Summing over these vectors only, we thus obtain the colouring polynomial $P_{G}^{x}(K)$. To calculate the total sum we use again the fact that the right-action of $G^{\prime}$ on $\tilde{Q}$ is transitive. Hence for every $q \in Q$ there exists $g \in G^{\prime}$ such that $s(q)^{g}=(x, 1)$. The action of $g$ induces a bijection between the set of basis vectors $\{s(q)\} \times B^{n-1}$ and $\{(x, 1)\} \times B^{n-1}$. Since the preceding trace calculation is $G^{\prime}$-invariant, each vector $v \in\{s(q)\} \times B^{n-1}$ contributes $P_{G}^{x}(K)$ to the trace. In total we obtain $\tilde{F}_{Q}=P_{G}^{x} \cdot|Q|$, as claimed.

4D. Concluding remarks. It follows from our construction that $\tilde{c}_{Q}$ is a deformation of the Yang-Baxter operator $c_{Q}$. More precisely we have $\tilde{c}_{Q}(a \otimes b)=$ $\lambda(a, b) \cdot c_{Q}(a, b)$ for all $a, b \in Q$ with a suitable map $\lambda: Q \times Q \rightarrow \Lambda$. Our 
construction via quandle coverings and central extensions provides a geometric interpretation in terms of meridian-longitude information. This interpretation carries through all steps of our construction, which finally allows us to interpret the resulting Yang-Baxter invariant as a colouring polynomial.

Conversely, it is natural to consider the ansatz $\tilde{c}_{Q}(a \otimes b)=\lambda(a, b) \cdot c_{Q}(a, b)$ and to ask which $\lambda$ turn $\tilde{c}_{Q}$ into a Yang-Baxter operator. This idea can, though in a restricted form, already be found in [Freyd and Yetter 1989, Thm.4.2.6]. A direct calculation shows that $\tilde{c}_{Q}$ is a Yang-Baxter operator if and only if $\lambda$ is a 2 -cocycle in the sense of quandle cohomology. Moreover, two such deformations will be equivalent if the cocycles differ by a coboundary. This observation has been worked out by M. Graña [2002], who independently proved that quandle 2-cocycle state-sum invariants are Yang-Baxter invariants.

\section{Acknowledgements}

The author thanks the anonymous referee for a careful reading and numerous helpful comments. The results of Section 2 were part of the author's Ph.D. thesis [Eisermann 2000a], which was financially supported by the Deutsche Forschungsgemeinschaft through the Graduiertenkolleg Mathematik at the University of Bonn. Sections 3 and 4 were elaborated while the author held a postdoc position at the École Normale Supérieure de Lyon, whose hospitality is gratefully acknowledged.

\section{References}

[Artin 1947] E. Artin, "Theory of braids", Ann. of Math. (2) 48 (1947), 101-126. MR 8,367a Zbl 0030.17703

[Bar-Natan 1995] D. Bar-Natan, “On the Vassiliev knot invariants”, Topology 34:2 (1995), 423-472. MR 97d:57004 Zbl 0898.57001

[Birman 1974] J. S. Birman, Braids, links, and mapping class groups, Annals of Mathematics Studies 82, Princeton University Press, Princeton, N.J., 1974. MR 51 \#11477 Zbl 0305.57013

[Brieskorn 1988] E. Brieskorn, "Automorphic sets and braids and singularities", pp. 45-115 in Braids (Santa Cruz, CA, 1986), edited by J. S. Birman and A. Libgober, Contemp. Math. 78, Amer. Math. Soc., Providence, RI, 1988. MR 90a:32024 Zbl 0716.20017

[Brown 1982] K. S. Brown, Cohomology of groups, Graduate Texts in Mathematics 87, Springer, New York, 1982. Corrected reprint, 1994. MR 96a:20072 Zbl 0584.20036

[Burde and Zieschang 1985] G. Burde and H. Zieschang, Knots, Studies in Mathematics 5, de Gruyter, Berlin, 1985. MR 87b:57004 Zbl 0568.57001

[Carter et al. 1999] J. S. Carter, D. Jelsovsky, S. Kamada, L. Langford, and M. Saito, "State-sum invariants of knotted curves and surfaces from quandle cohomology", Electron. Res. Announc. Amer. Math. Soc. 5 (1999), 146-156. MR 2002c:57014 Zbl 0995.57004

[Carter et al. 2003a] J. S. Carter, M. Elhamdadi, M. A. Nikiforou, and M. Saito, "Extensions of quandles and cocycle knot invariants", J. Knot Theory Ramifications 12:6 (2003), 725-738. MR 2004g:57020 Zbl 1049.57008 
[Carter et al. 2003b] J. S. Carter, D. Jelsovsky, S. Kamada, L. Langford, and M. Saito, "Quandle cohomology and state-sum invariants of knotted curves and surfaces", Trans. Amer. Math. Soc. 355:10 (2003), 3947-3989. MR 2005b:57048 Zbl 1028.57003

[Conway 1970] J. H. Conway, "An enumeration of knots and links, and some of their algebraic properties", pp. 329-358 in Computational Problems in Abstract Algebra (Proc. Conf., Oxford, 1967), Pergamon, Oxford, 1970. MR 41 \#2661 Zbl 0202.54703

[Conway et al. 1985] J. H. Conway, R. T. Curtis, S. P. Norton, R. A. Parker, and R. A. Wilson, Atlas of finite groups: Maximal subgroups and ordinary characters for simple groups, Oxford University Press, Eynsham, 1985. MR 88g:20025 Zbl 0568.20001

[Crowell and Fox 1963] R. H. Crowell and R. H. Fox, Introduction to knot theory, Ginn and Co., Boston, 1963. MR 26 \#4348 Zbl 0126.39105

[Dehn 1914] M. Dehn, “Die beiden Kleeblattschlingen”, Math. Ann. 75:3 (1914), 402-413. English translation in [Dehn 1987]. MR 1511799

[Dehn 1987] M. Dehn, Papers on group theory and topology, Springer, New York, 1987. Translated and edited by John Stillwell. MR 88d:01041

[Drinfel'd 1987] V. G. Drinfel'd, “Quantum groups”, pp. 798-820 in Proceedings of the International Congress of Mathematicians (Berkeley, 1986), vol. 1, Amer. Math. Soc., Providence, RI, 1987. MR 89f: 17017

[Eisermann 2000a] M. Eisermann, Knotengruppen-Darstellungen und Invarianten von endlichem Typ, Bonner Mathematische Schriften 327, Universität Bonn Mathematisches Institut, Bonn, 2000. (Author's dissertation). MR 2003g:57014 Zbl 0948.57008

[Eisermann 2000b] M. Eisermann, "The number of knot group representations is not a Vassiliev invariant", Proc. Amer. Math. Soc. 128:5 (2000), 1555-1561. MR 2000j:57009 Zbl 0939.57007

[Eisermann 2003] M. Eisermann, "Homological characterization of the unknot", J. Pure Appl. Algebra 177:2 (2003), 131-157. MR 2003j:57009 Zbl 1013.57002

[Eisermann 2005] M. Eisermann, "Yang-Baxter deformations of quandles and racks", Algebr. Geom. Topol. 5 (2005), 537-562. MR 2006b:17023 Zbl 02221853

[Fenn and Rourke 1992] R. Fenn and C. Rourke, "Racks and links in codimension two", J. Knot Theory Ramifications 1:4 (1992), 343-406. MR 94e:57006 Zbl 0787.57003

[Fox 1962] R. H. Fox, "A quick trip through knot theory", pp. 120-167 in Topology of 3-manifolds and related topics, Prentice-Hall, Englewood Cliffs, NJ, 1962. MR 25 \#3522

[Fox 1970] R. H. Fox, "Metacyclic invariants of knots and links", Canad. J. Math. 22 (1970), 193201. MR 41 \#6197 Zbl 0195.54002

[Freyd and Yetter 1989] P. J. Freyd and D. N. Yetter, "Braided compact closed categories with applications to low-dimensional topology", Adv. Math. 77:2 (1989), 156-182. MR 91c:57019 Zbl 0679.57003

[GAP 2006] GAP: Groups, Algorithms, and Programming, version 4.4.9, The GAP Group, 2006, Available at http://www.gap-system.org.

[González-Acuña 1975] F. González-Acuña, "Homomorphs of knot groups", Ann. of Math. (2) 102:2 (1975), 373-377. MR 52 \#576 Zbl 0323.57010

[Gordon and Luecke 1989] C. M. Gordon and J. Luecke, "Knots are determined by their complements", J. Amer. Math. Soc. 2:2 (1989), 371-415. MR 90a:57006a Zbl 0672.57009

[Graña 2002] M. Graña, "Quandle knot invariants are quantum knot invariants", J. Knot Theory Ramifications 11:5 (2002), 673-681. MR 2003e:57019 Zbl 1027.57014 
[Johnson 1980] D. Johnson, "Homomorphs of knot groups", Proc. Amer. Math. Soc. 78:1 (1980), 135-138. MR 80j:57004 Zbl 0435.57003

[Johnson and Livingston 1989] D. Johnson and C. Livingston, "Peripherally specified homomorphs of knot groups", Trans. Amer. Math. Soc. 311:1 (1989), 135-146. MR 89f:57006 Zbl 0662.57003

[Joyce 1982] D. Joyce, "A classifying invariant of knots, the knot quandle", J. Pure Appl. Algebra 23:1 (1982), 37-65. MR 83m:57007 Zbl 0474.57003

[Kassel 1995] C. Kassel, Quantum groups, Graduate Texts in Mathematics 155, Springer, New York, 1995. MR 96e:17041 Zbl 0808.17003

[Kassel et al. 1997] C. Kassel, M. Rosso, and V. Turaev, Quantum groups and knot invariants, Panoramas et Synthèses 5, Société Math. de France, Paris, 1997. MR 99b:57011 Zbl 0878.17013

[Kauffman 1987] L. H. Kauffman, "State models and the Jones polynomial", Topology 26:3 (1987), 395-407. MR 88f:57006 Zbl 0622.57004

[Kauffman 2001] L. H. Kauffman, Knots and physics, Third ed., Series on Knots and Everything 1, World Scientific, River Edge, NJ, 2001. MR 2002h:57012 Zbl 1057.57001

[Kuperberg 1996] G. Kuperberg, "Detecting knot invertibility", J. Knot Theory Ramifications 5:2 (1996), 173-181. MR 97h:57018 Zbl 0858.57011

[Lickorish 1997] W. B. R. Lickorish, An introduction to knot theory, Graduate Texts in Mathematics 175, Springer, New York, 1997. MR 98f:57015 Zbl 0886.57001

[Matveev 1982] S. V. Matveev, "Distributive groupoids in knot theory", Mat. Sb. (N.S.) 119 (1982), 78-88. In Russian; translated in Math. USSR Sb. 47 (1984), 73-83. MR 84e:57008 Zbl 0523.57006

[Neuwirth 1965] L. P. Neuwirth, Knot groups, Annals of Mathematics Studies 56, Princeton University Press, Princeton, N.J., 1965. MR 31 \#734 Zbl 0184.48903

[Riley 1971] R. Riley, "Homomorphisms of knot groups on finite groups", Math. Comp. 25 (1971), 603-619. Addendum in 25 (1971), no. 115, microfiche suppl. A-B. MR 45 \#4399 Zbl 0224.55003

[Thurston 1982] W. P. Thurston, "Three-dimensional manifolds, Kleinian groups and hyperbolic geometry”, Bull. Amer. Math. Soc. (N.S.) 6:3 (1982), 357-381. MR 83h:57019 Zbl 0496.57005

[Trotter 1963] H. F. Trotter, "Non-invertible knots exist", Topology 2 (1963), 275-280. MR 28 \#1618 Zbl 0136.21203

[Waldhausen 1968] F. Waldhausen, "On irreducible 3-manifolds which are sufficiently large", Ann. of Math. (2) 87 (1968), 56-88. MR 36 \#7146 Zbl 0157.30603

[Whitten 1987] W. Whitten, "Knot complements and groups", Topology 26:1 (1987), 41-44. MR 88f:57014 Zbl 0607.57004

Received July 24, 2004. Revised July 25, 2007.

\section{MiCHAEL EISERMANN \\ INSTITUT FOURIER \\ UNIVERSITÉ GRENOBLE I \\ 100 RUE DES MATHS, BP 74 \\ 38402 ST MARTIN D'HÈRES \\ FRANCE}

Michael.Eisermann@ujf-grenoble.fr

http://www-fourier.ujf-grenoble.fr/ eiserm 


\title{
PACIFIC JOURNAL OF MATHEMATICS
}

\author{
msp.org/pjm
}

Founded in 1951 by E. F. Beckenbach (1906-1982) and F. Wolf (1904-1989)

\section{EDITORS}

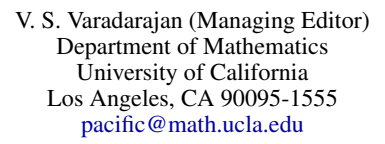

\author{
Vyjayanthi Chari \\ Department of Mathematics \\ University of California \\ Riverside, CA 92521-0135 \\ chari@math.ucr.edu \\ Robert Finn \\ Department of Mathematics \\ Stanford University \\ Stanford, CA 94305-2125 \\ finn@math.stanford.edu \\ Kefeng Liu \\ Department of Mathematics \\ University of California \\ Los Angeles, CA 90095-1555 \\ liu@math.ucla.edu
}

Paulo Ney de Souza, Production Manager

ACADEMIA SINICA, TAIPEI

CALIFORNIA INST. OF TECHNOLOGY

INST. DE MATEMÁTICA PURA E APLICADA

KEIO UNIVERSITY

MATH. SCIENCES RESEARCH INSTITUTE

NEW MEXICO STATE UNIV.

OREGON STATE UNIV.

PEKING UNIVERSITY

STANFORD UNIVERSITY

\author{
Darren Long \\ Department of Mathematics \\ University of California \\ Santa Barbara, CA 93106-3080 \\ long@math.ucsb.edu \\ Jiang-Hua Lu \\ Department of Mathematics \\ The University of Hong Kong \\ Pokfulam Rd., Hong Kong \\ jhlu@maths.hku.hk \\ Alexander Merkurjev \\ Department of Mathematics \\ University of California \\ Los Angeles, CA 90095-1555 \\ merkurev@math.ucla.edu
}

\section{PRODUCTION}

Silvio Levy, Senior Production Editor

\section{SUPPORTING INSTITUTIONS}

UNIVERSIDAD DE LOS ANDES
UNIV. OF ARIZONA
UNIV. OF BRITISH COLUMBIA
UNIV. OF CALIFORNIA, BERKELEY
UNIV. OF CALIFORNIA, DAVIS
UNIV. OF CALIFORNIA, IRVINE
UNIV. OF CALIFORNIA, LOS ANGELES
UNIV. OF CALIFORNIA, RIVERSIDE
UNIV. OF CALIFORNIA, SAN DIEGO
UNIV. OF CALIF., SANTA BARBARA

Sorin Popa

Department of Mathematics

University of California

Los Angeles, CA 90095-1555

popa@math.ucla.edu

\section{Jie Qing}

Department of Mathematics University of California Santa Cruz, CA 95064 qing@cats.ucsc.edu

Jonathan Rogawski

Department of Mathematics

University of California

Los Angeles, CA 90095-1555 jonr@math.ucla.edu

Alexandru Scorpan, Production Editor

\author{
UNIV. OF CALIF., SANTA CRUZ \\ UNIV. OF HAWAII \\ UNIV. OF MONTANA \\ UNIV. OF NEVADA, RENO \\ UNIV. OF OREGON \\ UNIV. OF SOUTHERN CALIFORNIA \\ UNIV. OF UTAH \\ UNIV. OF WASHINGTON \\ WASHINGTON STATE UNIVERSITY
}

These supporting institutions contribute to the cost of publication of this Journal, but they are not owners or publishers and have no responsibility for its contents or policies.

See inside back cover or msp.org/pjm for submission instructions.

Regular subscription rate for 2007: \$425.00 a year (10 issues). Special rate: $\$ 212.50$ a year to individual members of supporting institutions.

Subscriptions, requests for back issues and changes of subscribers address should be sent to Pacific Journal of Mathematics, P.O. Box 4163, Berkeley, CA 94704-0163, U.S.A. The Pacific Journal of Mathematics is indexed by Mathematical Reviews, Zentralblatt MATH, PASCAL CNRS Index, Referativnyi Zhurnal, Current Mathematical Publications and Web of Knowledge (Science Citation Index).

The Pacific Journal of Mathematics (ISSN 0030-8730) at the University of California, c/o Department of Mathematics, 798 Evans Hall \#3840, Berkeley, CA 94720-3840, is published twelve times a year. Periodical rate postage paid at Berkeley, CA 94704, and additional mailing offices. POSTMASTER: send address changes to Pacific Journal of Mathematics, P.O. Box 4163, Berkeley, CA 94704-0163.

PJM peer review and production are managed by EditFLOW ${ }^{\circledR}$ from Mathematical Sciences Publishers.

PUBLISHED BY

\section{D. mathematical sciences publishers \\ nonprofit scientific publishing}

http://msp.org/

(C) 2014 Mathematical Sciences Publishers 


\section{PACIFIC JOURNAL OF MATHEMATICS}

Volume $231 \quad$ No. $2 \quad$ June 2007

The Euclidean rank of Hilbert geometries

OLIVER BLETZ-SIEBERT and THOMAS FOERTSCH

A volumish theorem for the Jones polynomial of alternating knots

279

OLIVER T. DASBACH and XIAO-SONG LIN

On the local Nirenberg problem for the $Q$-curvatures

293

PHILIPPE DELANOË and FrÉDÉRIC ROBERT

Knot colouring polynomials

305

MICHAEL EISERMANN

Some new simple modular Lie superalgebras

Alberto ElduQue

Subfactors from braided $C^{*}$ tensor categories

JULIANA ERLIJMAN and HANS WENZL

An elementary, explicit, proof of the existence of Quot schemes of points

401

Trond Stølen Gustavsen, Dan Laksov and Roy MiKael

SKJELNES

Symplectic energy and Lagrangian intersection under Legendrian

deformations

HAI-LONG HER

Harmonic nets in metric spaces

JÜRGEN JOST and LEONARD TODJIHOUNDE

The quantitative Hopf theorem and filling volume estimates from below

LUOFEI LIU

On the variation of a series on Teichmüller space

GREg McShane

On the geometric and the algebraic rank of graph manifolds 